\title{
Pacific
}

Journal of

Mathematics

\section{NUMERICAL STUDY OF UNBOUNDED CAPILLARY SURFACES}

YASUnORi AOKI AND HANS De STERCK 


\title{
NUMERICAL STUDY OF UNBOUNDED CAPILLARY SURFACES
}

\author{
YASUNORi AOKI AND HANS De STERCK
}

\begin{abstract}
Unbounded capillary surfaces in domains with a sharp corner or a cusp are studied. It is shown how numerical study using a proposed computational methodology leads to two new conjectures for open problems on the asymptotic behavior of capillary surfaces in domains with a cusp. The numerical methodology contains two simple but important ingredients, a change of variable and a change of coordinates, which are inspired by known asymptotic approximations for unbounded capillary surfaces. These ingredients are combined with the finite volume element or Galerkin finite element methods. Extensive numerical tests show that the proposed computational methodology leads to a global approximation method for singular solutions of the Laplace-Young equation that recovers the proper asymptotic behavior at the singular point, is more accurate and has better convergence properties than numerical methods considered for singular capillary surfaces before. Using this computational methodology, two open problems on the asymptotic behavior of capillary surfaces in domains with a cusp are studied numerically, leading to two conjectures that may guide future analytical work on these open problems.
\end{abstract}

\section{Introduction}

The mathematical analysis of unbounded capillary surfaces is most often done by asymptotic analysis (see [Concus and Finn 1970; 1974; 1989; Miersemann 1993; King et al. 1999; Norbury et al. 2005; Scholz 2001; 2004; Aoki 2007; Aoki and Siegel 2012]). Results for unbounded capillary surfaces in domains with sharp corners have been known for many years, and recent work of Aoki and Siegel [2012] on singular capillary surfaces in domains with a cusp fills almost all the gaps that still existed for the cusp case, though a few open problems remain. Since asymptotic analysis is a local analysis, asymptotic approximations are valid only in a sufficiently small domain near the singularity. It is also not easy to determine the precise region of validity of the asymptotic analysis results. In applications, global

MSC2010: 35J25, 65N30, 76B45, 35J75.

Keywords: singularity, asymptotic analysis, nonlinear elliptic PDE, Laplace-Young equation, finite element method. 
approximations for the singular capillary surfaces that are valid in the whole domain are desirable, and such approximations cannot be provided by asymptotic analysis.

In this paper, our aim is to construct globally valid approximations of singular capillary surfaces which exhibit the proper asymptotic behavior at the singular point while also being valid away from the singularity. We do so by introducing a computational methodology for singular capillary surfaces. A second aim of this paper is to investigate two open problems on asymptotic behavior of capillary surfaces in domains with a cusp. We investigate these open problems using the proposed numerical methodology, which leads to two conjectures that may guide future analytical work on these open problems.

Our computational methodology starts from the finite volume element method (FVEM) [Bank and Rose 1987; Aoki and De Sterck 2011] or the Galerkin finite element method (FEM) [Strang and Fix 1973; Brenner and Scott 1994]. However, it is widely known (see [Grisvard 1985; Strang and Fix 1973; Aoki and De Sterck 2011]) that a lack of smoothness in the solution can spoil the accuracy of approximations of finite element type; hence it can be expected that standard finite element approximations cannot accurately approximate the unbounded singularity. There are about a half dozen published papers on numerical solutions of the LaplaceYoung equation [Nigro et al. 2000; Hornung and Mittelmann 1990; Polevikov 2004; Polevikov 1999; Scott et al. 2005]. However, with the exception of the paper by Scott et al. [2005] they do not consider unbounded singular solutions. Scott et al. use the finite volume element method to approximate solutions of the Laplace-Young equation, and one of their model problems is a corner problem with unbounded singularity. Our proposed methodology enhances their approach in two important ways, leading to much more accurate and informative results, as shown in our numerical results section.

Instead of directly approximating the solution with a standard finite element expansion, our idea is to incorporate knowledge obtained from asymptotic analysis into the finite element approximation, in order to avoid inaccuracies introduced by the singularity. Roughly speaking, we first change the variable based on the known asymptotic order of the solution so that the new unknown function is bounded. (Though it is bounded, it can still be discontinuous at the location of the original singularity.) Inspired by knowledge of the leading-order term of the asymptotic series solution, we change the coordinate system so that the unknown function is smooth with respect to the new coordinate variables. We then finally approximate the smooth bounded new unknown function with respect to the new coordinate variables, using the finite volume element method or the Galerkin finite element method.

We verify the accuracy of this numerical methodology by comparing the numerical solution with known asymptotic series approximations, and by conducting 
numerical convergence studies. We first show that the numerical solutions we obtain have the proper singular behavior by comparing numerical solutions of the Laplace-Young equation with known asymptotic series approximations. Then we conduct numerical convergence studies to show that the numerical approximation is a globally valid approximation. In order to conduct numerical convergence studies, we need model problems with known closed-form solutions. Though there is no known unbounded closed-form solution of the Laplace-Young equation, a few closed-form solutions are known for the steep slope approximation of the LaplaceYoung equation [King et al. 1999; Aoki 2007] (we shall refer to this PDE as the asymptotic Laplace-Young equation). It is known that these solutions have the same asymptotic behavior as the solution of the original problem, so we conduct the convergence study using the asymptotic Laplace-Young equation.

Using the proposed numerical methodology for computing singular solutions of the Laplace-Young equation, we investigate a few open problems of singular behavior of the Laplace-Young equation in a cusp domain. Aoki and Siegel [2012] considered the solution behavior for all possible cusp domains, attempting to generalize the results of Scholz [2004]. However, there are still a few cases that remain open. Using our computational methodology, we numerically investigate these cases and make conjectures based on the numerical approximations.

The paper proceeds as follows. In Section 2 we describe the Laplace-Young boundary value problem of interest and its asymptotic variant in domains with a sharp corner or a cusp. Section 3 describes the proposed numerical methodology for computing accurate global numerical approximations of unbounded capillary surfaces in these types of domains, and Section 4 gives extensive numerical results verifying the accuracy and convergence of the numerical methods. Section 5 presents conjectures for two open cases on asymptotic behavior of capillary surfaces in a domain with a cusp, motivated by numerical solutions for these open cases using the proposed numerical methodology. Finally, conclusions are formulated in Section 6.

\section{The boundary value problem}

In this section we first formulate the Laplace-Young boundary value problem, and describe the asymptotic behavior of its solutions in domains with a corner or a cusp and the function spaces these solutions belong to. We state some open problems on asymptotic behavior for a domain with an osculatory cusp and a cusp with infinite curvature, and define model problems that will be used in numerical tests. We then describe the asymptotic Laplace-Young equation and its known closed-form solutions on domains with a corner or a cusp, which are used to formulate additional numerical model problems. 

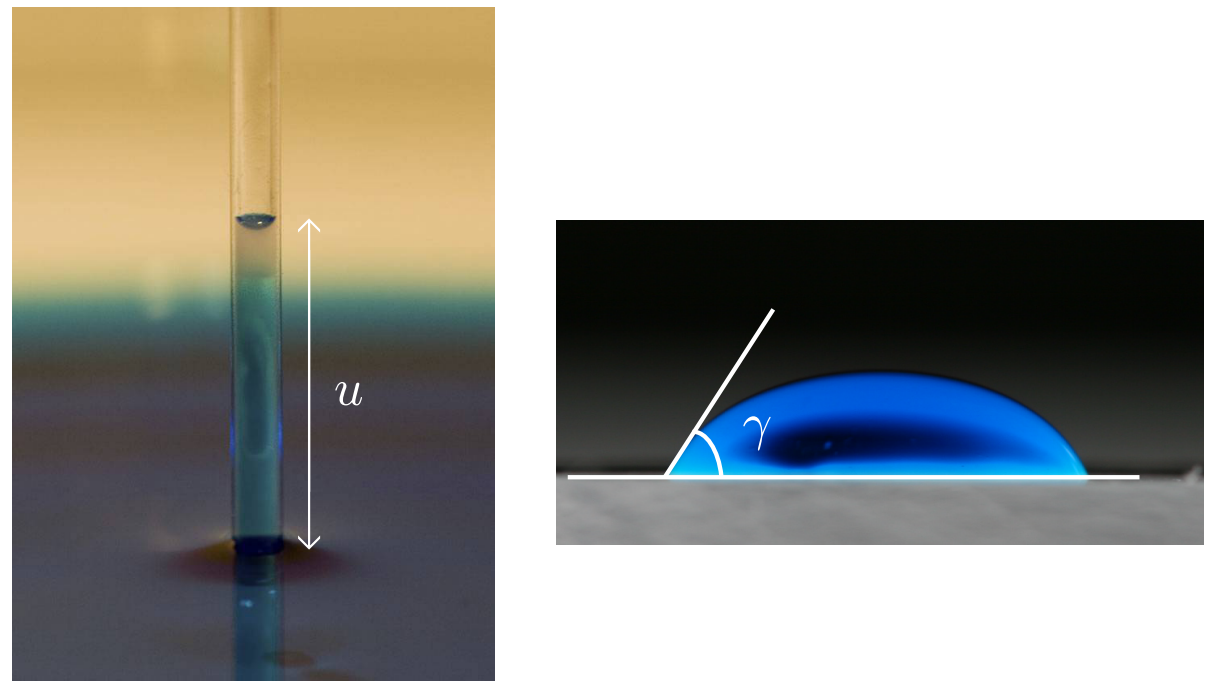

Figure 1. Two photos of capillarity experiments, indicating the capillary surface height $u$ and the contact angle $\gamma$.

2.1. Laplace-Young boundary value problem. This problem originates from observations of Laplace in 1806 and Young in 1805 that "the height of the liquid is proportional to its mean curvature" and "the angle of the contact between the solid and liquid only depends on their material." Gauss showed in 1830 that the Laplace-Young PDE is in fact the Euler-Lagrange equation of the surface energy functional. Thus the solution of the Laplace-Young boundary value problem gives the shape of the liquid surface that minimizes the surface energy, in a nonvanishing downward gravity field, and hence the Laplace-Young boundary value problem is a mathematical model for a liquid surface at equilibrium when the gravity is present. We refer the reader to Section 1.4 of a book by Finn [1986] for detailed discussion of the derivation of the Laplace-Young boundary value problem. Figure 1 shows photos of capillarity experiments, indicating the capillary surface height $u$ and the contact angle $\gamma$.

Let $\Omega$ be an unbounded open domain as in Figure 2 with boundaries $\partial \Omega_{1}$ and $\partial \Omega_{2}$ described by functions $f_{1}(x)$ and $f_{2}(x)$, and let $u \in C^{2}(\Omega)$ be the height of the capillary surface that satisfies the following boundary value problem (the Laplace-Young boundary value problem) on this domain:

$$
\begin{array}{ll}
\nabla \cdot T(u)=\kappa u & \text { in } \Omega, \\
\vec{v}_{1} \cdot T(u)=\cos \gamma_{1} & \text { on } \partial \Omega_{1}, \\
\vec{v}_{2} \cdot T(u)=\cos \gamma_{2} & \text { on } \partial \Omega_{2},
\end{array}
$$



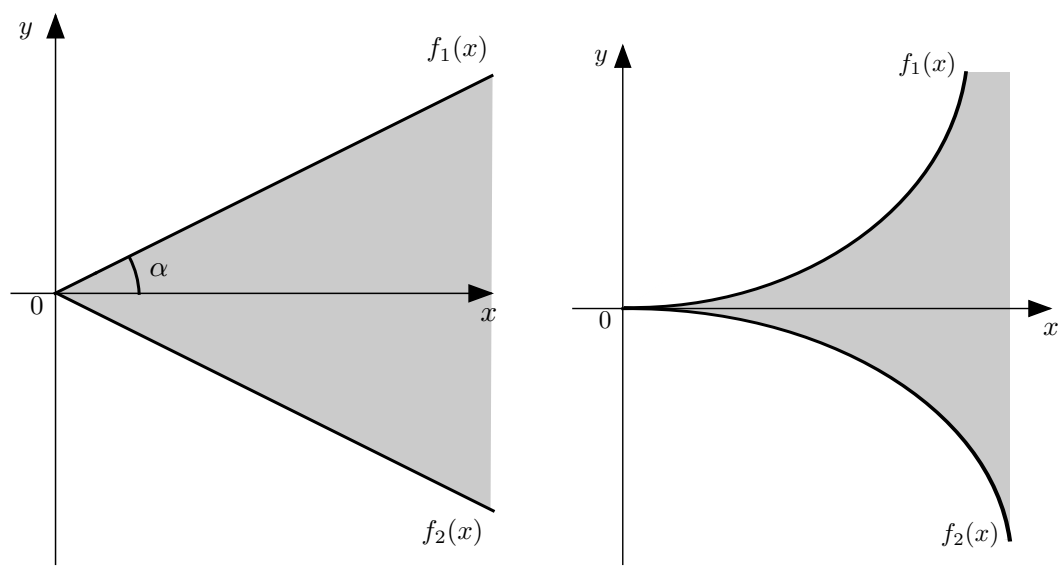

Figure 2. Unbounded domains. Left: a corner domain. Right: a cusp domain.

with

$\kappa$ : capillarity constant (we assume $\kappa>0$ ),

$\vec{v}_{1}, \vec{v}_{2}$ : exterior unit normal vectors on the boundaries $\partial \Omega_{1}$ and $\partial \Omega_{2}$,

$\gamma_{1}, \gamma_{2}:$ contact angles

and

$$
T(u)=\frac{\nabla u}{\sqrt{1+|\nabla u|^{2}}} .
$$

Note that the capillarity constant $\kappa$ can be normalized by rescaling $x, y$ and $u$ when $\kappa>0$. In the following sections we let $\kappa=1$. The open domain $\Omega$ and boundaries $\partial \Omega_{1}$ and $\partial \Omega_{2}$ are defined more specifically as follows:

$$
\begin{aligned}
\Omega & =\left\{(x, y) \in \mathbb{R}^{2}: 0<x, f_{2}(x)<y<f_{1}(x)\right\}, \\
\partial \Omega_{1} & =\left\{(x, y) \in \mathbb{R}^{2}: 0<x, y=f_{1}(x)\right\}, \\
\partial \Omega_{2} & =\left\{(x, y) \in \mathbb{R}^{2}: 0<x, y=f_{2}(x)\right\},
\end{aligned}
$$

with

$$
\begin{aligned}
f_{1}(x), f_{2}(x) & \in C^{3}(0, \infty), \\
f_{1}(x) & >f_{2}(x) \text { for } x>0,
\end{aligned}
$$

$$
\begin{aligned}
\lim _{x \rightarrow 0^{+}} f_{1}(x)=\lim _{x \rightarrow 0^{+}} f_{2}(x)=0, \\
\lim _{x \rightarrow 0^{+}} f_{1}^{\prime}(x) \neq \infty \neq \lim _{x \rightarrow 0^{+}} f_{2}^{\prime}(x) .
\end{aligned}
$$


For simplicity of discussion we focus on the two specific types of domains depicted in Figure 2: a corner domain, defined as

$$
f_{1}(x)=x \tan \alpha \quad \text { and } \quad f_{2}(x)=-x \tan \alpha, \quad \text { where } 0<\alpha<\pi / 2,
$$

and a cusp domain, defined as

$$
\lim _{x \rightarrow 0^{+}} f_{1}^{\prime}(x)=0 \quad \text { and } \quad \lim _{x \rightarrow 0^{+}} f_{2}^{\prime}(x)=0 .
$$

2.1.1. Asymptotic behavior. It is known that the solution of the Laplace-Young boundary value problem in a corner domain is unbounded at $(0,0)$ if $\gamma_{1}+\gamma_{2}+2 \alpha<\pi$ (see [Finn 1986]). Also, it can be shown that if $\gamma_{1}+\gamma_{2} \neq \pi$ the solution of the boundary value problem in a cusp domain is unbounded at $(0,0)$ (see [Scholz 2004; Aoki and Siegel 2012]). In addition, the following asymptotic behaviors are known.

Corner domain with $\gamma_{1}+\gamma_{2}+2 \alpha<\pi$ (see [Concus and Finn 1970; Miersemann 1993] for a proof): If $\gamma_{1}=\gamma_{2}=\gamma$ and $\gamma+\alpha<\pi / 2$, then the solution of the boundary value problem in the corner domain has the following asymptotic behavior:

$$
u(r, \theta)=\frac{\cos \theta-\sqrt{k^{2}-\sin ^{2} \theta}}{k r}+O\left(r^{3}\right) \quad \text { as } r \rightarrow 0,
$$

where

$$
\begin{gathered}
(r, \theta): \text { polar coordinate variables, } \\
k=\frac{\sin \alpha}{\cos \gamma} .
\end{gathered}
$$

More formally, we can write that there exist constants $r_{o}$ and $M$ such that

$$
\left|u-\frac{\cos \theta-\sqrt{k^{2}-\sin ^{2} \theta}}{k r}\right|<M r^{3} \text { for } 0<r<r_{o} .
$$

This gives the following bounds for the solution $u$ :

$$
\frac{\cos \theta-\sqrt{k^{2}-\sin ^{2} \theta}}{k r}-M r^{3}<u<\frac{\cos \theta-\sqrt{k^{2}-\sin ^{2} \theta}}{k r}+M r^{3}
$$

$$
\text { for } 0<r<r_{0} \text {. }
$$

The proof for the asymptotic relation (8) only provides the existence of these two constants and does not give any estimate of their size. Thus, even though (8) shows that the asymptotic approximation becomes more and more accurate as we get closer to the singularity, it does not give any quantitative description of the approximation error. 
Also, it is easy to show from (9) that there exist positive constants $M^{+}, M^{-}$, and $x_{o}$ such that

$$
\frac{M^{-}}{f_{1}(x)-f_{2}(x)}<u<\frac{M^{+}}{f_{1}(x)-f_{2}(x)} \text { for } 0<x<x_{o} .
$$

Cusp domain with $\gamma_{1}+\gamma_{2} \neq \pi$ (see [Aoki and Siegel 2012] for a proof): An unbounded solution of the boundary value problem in a cusp domain has the asymptotic behavior

$$
u(x, y)=\frac{\cos \gamma_{1}+\cos \gamma_{2}}{f_{1}(x)-f_{2}(x)}+O\left(\frac{f_{1}^{\prime}(x)-f_{2}^{\prime}(x)}{f_{1}(x)-f_{2}(x)}\right) \quad \text { as } x \rightarrow 0^{+}
$$

if $\gamma_{1}+\gamma_{2} \neq \pi$ and the boundary functions $f_{1}(x)$ and $f_{2}(x)$ satisfy the asymptotic relations

$$
\begin{aligned}
f_{1}(x)-f_{2}(x) & =o\left(f_{1}^{\prime}(x)-f_{2}^{\prime}(x)\right), \\
\frac{f_{1}^{\prime \prime}(x)-f_{2}^{\prime \prime}(x)}{f_{1}(x)-f_{2}(x)} & =\alpha \frac{\left(f_{1}^{\prime}(x)-f_{2}^{\prime}(x)\right)^{2}}{\left(f_{1}(x)-f_{2}(x)\right)^{2}}+o\left(\frac{\left(f_{1}^{\prime}(x)-f_{2}^{\prime}(x)\right)^{2}}{\left(f_{1}(x)-f_{2}(x)\right)^{2}}\right), \\
\frac{f_{1}^{\prime \prime \prime}(x)-f_{2}^{\prime \prime \prime}(x)}{f_{1}^{\prime}(x)-f_{2}^{\prime}(x)} & =O\left(\frac{\left(f_{1}^{\prime}(x)-f_{2}^{\prime}(x)\right)^{2}}{\left(f_{1}(x)-f_{2}(x)\right)^{2}}\right), \\
f_{1}^{\prime}(x)+f_{2}^{\prime}(x) & =\delta\left(f_{1}^{\prime}(x)-f_{2}^{\prime}(x)\right)+o\left(f_{1}^{\prime}(x)-f_{2}^{\prime}(x)\right), \\
f_{1}^{\prime \prime}(x)+f_{2}^{\prime \prime}(x) & =O\left(f_{1}^{\prime \prime}(x)-f_{2}^{\prime \prime}(x)\right)
\end{aligned}
$$

as $x \rightarrow 0$, where $\alpha, \delta \in \mathbb{R}$.

Note that most boundary functions forming cusp domains satisfy the asymptotic conditions (12)-(14). One known exception is when the boundary functions forming a cusp are osculatory at the cusp. Curves are said to be osculatory if they intersect and share the tangent line and the osculating circle at the intersection point. Again it follows from (11) that there exist positive constants $M^{+}, M^{-}$, and $x_{o}$ such that

$$
\frac{M^{-}}{f_{1}(x)-f_{2}(x)}<u<\frac{M^{+}}{f_{1}(x)-f_{2}(x)} \text { for } 0<x<x_{o} .
$$

2.1.2. Open problems. To the authors' knowledge there are two major open problems in the solution behavior of the Laplace-Young equation in a domain with a cusp. We summarize these open problems.

Problem 1: Osculatory cusp with nonsupplementary contact angles $\left(\gamma_{1}+\gamma_{2} \neq \pi\right)$ : An osculatory cusp is a cusp formed by two osculating curves. It is known that the solution is unbounded when $\gamma_{1}+\gamma_{2} \neq \pi$, but the asymptotic expansion from the previous section does not apply in the osculatory cusp case and remains an open problem. For example, the two boundary functions

$$
f_{1}(x)=x^{2}+x^{3} \quad \text { and } \quad f_{2}(x)=x^{2}-x^{3}
$$


form an osculatory cusp at the origin. The asymptotic orders of the sum and the difference of these boundary functions are different (i.e., $f_{1}(x)-f_{2}(x)=O\left(x^{3}\right)$ while $f_{1}(x)+f_{2}(x)=O\left(x^{2}\right)$ as $\left.x \rightarrow 0\right)$. Hence these choices of $f_{1}(x)$ and $f_{2}(x)$ do not satisfy the asymptotic relations (13)-(14), so that the leading-order asymptotic behavior of the solution at this cusp is unknown. The main reason why the proof for the leading-order asymptotic behavior could not be constructed for the osculatory cusp case is that the second-order term of the formal asymptotic series could not be found (see [Aoki and Siegel 2012] for details).

Problem 2: Infinite curvature cusp with supplementary contact angles $\left(\gamma_{1}+\gamma_{2}=\pi\right)$ : As was noted before, the solution of the Laplace-Young equation in a cusp domain is unbounded if $\gamma_{1}+\gamma_{2} \neq \pi$, but it is not necessarily true that the solution is bounded if $\gamma_{1}+\gamma_{2}=\pi$. Aoki and Siegel [2012] have shown that the solution is bounded if $\gamma_{1}+\gamma_{2}=\pi$ and the curvatures of the boundary functions are finite (i.e., $\lim _{x \rightarrow 0} f_{1}^{\prime \prime}(x) \neq \infty$ and $\left.\lim _{x \rightarrow 0} f_{2}^{\prime \prime}(x) \neq \infty\right)$. However, the nature of the solution for the case where the curvatures of one or both boundary functions are infinite is not known (e.g., $f_{1}(x)=x^{3 / 2}$ and $f_{2}(x)=-2 x^{3 / 2}$ ).

2.1.3. Model Problems 1 and 2. For the numerical experiments to be reported on below we consider the following model problems (henceforth, MPs).

Consider bounded open domains $\Omega$ as depicted in Figure 3. Let $u \in C^{2}(\Omega)$ be the height of the capillary surface that satisfies the following boundary value problem:

$$
\begin{array}{ll}
\nabla \cdot T(u)=u & \text { in } \Omega, \\
\vec{v}_{1} \cdot T(u)=\cos \gamma_{1} & \text { on } \partial \Omega_{1}, \\
\vec{v}_{2} \cdot T(u)=\cos \gamma_{2} & \text { on } \partial \Omega_{2}, \\
\vec{v}_{3} \cdot T(u)=0 & \text { on } \partial \Omega_{3},
\end{array}
$$
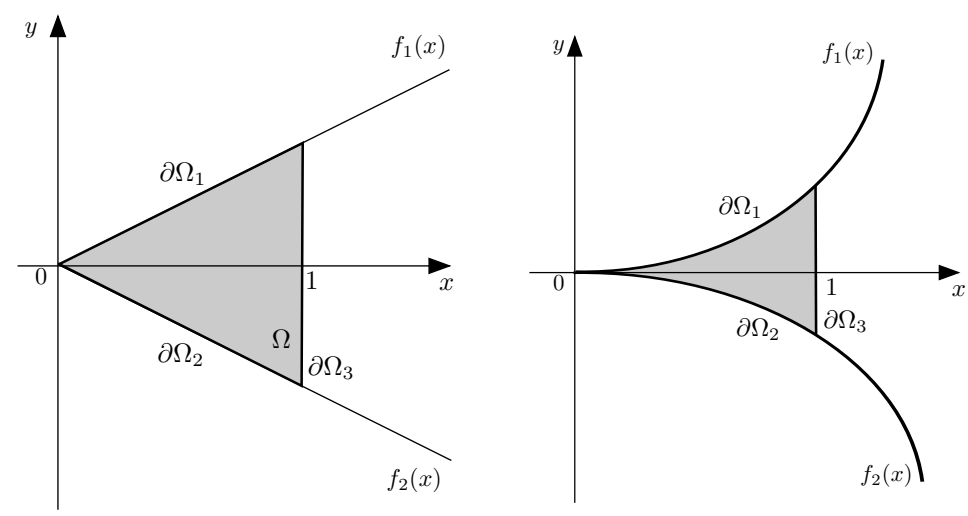

Figure 3. Computational domains for model problems with a corner and a cusp at $(0,0)$. Left: problems 1 and 3. Right: problems 2 and 4. 
with

$\vec{v}_{1}, \vec{v}_{2}, \vec{v}_{3}$ : exterior unit normal vectors on the boundaries $\partial \Omega_{1}, \partial \Omega_{2}$ and $\partial \Omega_{3}$, $\gamma_{1}, \gamma_{2}:$ contact angles.

The bounded open domain $\Omega$ and boundaries $\partial \Omega_{1,2,3}$ are defined more specifically as follows:

$$
\begin{aligned}
\Omega & =\left\{(x, y) \in \mathbb{R}^{2}: 0<x<1, f_{2}(x)<y<f_{1}(x)\right\}, \\
\partial \Omega_{1} & =\left\{(x, y) \in \mathbb{R}^{2}: 0<x<1, y=f_{1}(x)\right\}, \\
\partial \Omega_{2} & =\left\{(x, y) \in \mathbb{R}^{2}: 0<x<1, y=f_{2}(x)\right\}, \\
\partial \Omega_{3} & =\left\{(x, y) \in \mathbb{R}^{2}: x=1, f_{2}(1)<y<f_{1}(1)\right\} .
\end{aligned}
$$

The boundary functions and the contact angles are chosen for each model problem as tabulated in Tables 1 and 2. Although there are cases in which the behavior of bounded solutions in a corner domain has special interest (e.g., [Lancaster 2010]), in this paper we focus our attention on unbounded solutions in a corner domain.

These model problems are chosen so that the singularity may only occur at the corner or cusp at the origin, although there are three nonsmooth points on the boundary of the domain $\Omega$. Following immediately from the regularity result of Simon [1980], this implies that the solutions $u$ of these model problems are differentiable up to the boundary except at the origin, i.e., $u \in C^{1}(\bar{\Omega} \backslash\{(0,0)\})$. Also, the asymptotic behavior of the solution at the origin is known to be as stated in (8) for MP $1\left(\gamma_{1}+\gamma_{2}+2 \alpha<\pi\right.$, unbounded), and as in (11) for MPs $2 \mathrm{a}\left(\gamma_{1}+\gamma_{2} \neq \pi\right.$, unbounded) and $2 \mathrm{c}-1$ and $2 \mathrm{c}-3\left(\gamma_{1}+\gamma_{2} \neq \pi\right.$, unbounded $)$. The asymptotic expansions for MPs $2 \mathrm{~b}$ (osculatory cusp with nonsupplementary contact angles) are an open problem (but it is known that the solutions are unbounded). The asymptotic behavior of the solution of MP 2c-2 (infinite curvature cusp with supplementary contact angles) is an open problem.

2.1.4. Solution function spaces. It is interesting to discuss the function spaces where the solutions of MP 1 and MPs 2a, 2c-1, and 2c-3 reside.

\begin{tabular}{|cccccc|}
\hline Problem & $f_{1}(x)$ & $f_{2}(x)$ & $\gamma_{1}$ & $\gamma_{2}$ & \\
\hline $1-1$ & $x \tan (\pi / 7)$ & $-x \tan (\pi / 7)$ & $\pi / 6$ & $\pi / 6$ & Corner (unbounded) \\
$1-2$ & $x \tan (\pi / 7)$ & $-x \tan (\pi / 7)$ & $\pi / 4$ & $\pi / 4$ & Corner (unbounded) \\
$1-3$ & $x \tan (\pi / 7)$ & $-x \tan (\pi / 7)$ & $\pi / 3$ & $\pi / 3$ & Corner (unbounded) \\
\hline
\end{tabular}

Table 1. Model Problem 1: Laplace-Young equation in a domain with a corner. All three variants have $\alpha=\pi / 7$ and $\gamma_{1}+\gamma_{2}+2 \alpha<\pi$, resulting in solutions that are unbounded at $(0,0)$. 


\begin{tabular}{|cccccccc|}
\hline Problem & $f_{1}(x)$ & $f_{2}(x)$ & $\gamma_{1}$ & $\gamma_{2}$ & cusp & unbd? & open? \\
\hline $2 \mathrm{a}-1$ & $x^{2} / 6$ & $-x^{3} / 8$ & $\pi / 6$ & $\pi / 3$ & $\mathrm{R}$ & yes & no \\
$2 \mathrm{a}-2$ & $x^{3} / 6$ & $-x^{3} / 8$ & $\pi / 3$ & $\pi / 4$ & $\mathrm{R}$ & yes & no \\
$2 \mathrm{a}-3$ & $x^{5} / 6$ & $-x^{4} / 8$ & $\pi / 3$ & $\pi / 4$ & $\mathrm{R}$ & yes & no \\
$2 \mathrm{~b}-1$ & $\left(x^{2}+x^{3}\right) / 6$ & $\left(x^{2}-\frac{3}{4} x^{3}\right) / 6$ & $\pi / 3$ & $\pi / 4$ & $\mathrm{O}$ & yes & yes \\
$2 \mathrm{~b}-2$ & $\left(3 x^{2}+x^{3}\right) / 6$ & $\left(3 x^{2}-\frac{3}{4} x^{3}\right) / 6$ & $\pi / 3$ & $\pi / 4$ & $\mathrm{O}$ & yes & yes \\
$2 \mathrm{~b}-3$ & $x^{3 / 2}+x^{3} / 6$ & $x^{3 / 2}-\frac{3}{4} x^{3} / 6$ & $\pi / 3$ & $\pi / 4$ & $\mathrm{O}$ & yes & yes \\
$2 \mathrm{c}-1$ & $x^{3 / 2} / 6$ & $x^{3 / 2} / 8$ & $5 \pi / 6-\pi / 180$ & $\pi / 6$ & $\mathrm{IC}$ & yes & no \\
$2 \mathrm{c}-2$ & $x^{3 / 2} / 6$ & $x^{3 / 2} / 8$ & $5 \pi / 6$ & $\pi / 6$ & $\mathrm{IC}$ & $?$ & yes \\
$2 \mathrm{c}-3$ & $x^{3 / 2} / 6$ & $x^{3 / 2} / 8$ & $5 \pi / 6+\pi / 180$ & $\pi / 6$ & $\mathrm{IC}$ & yes & no \\
\hline
\end{tabular}

Table 2. Model Problem 2: Laplace-Young equation in a domain with a cusp. Variants 2a, 2c-1, and 2c-3 have unbounded solutions at $(0,0)$ and known asymptotic expansions. Variants $2 \mathrm{~b}$ also have unbounded solutions at $(0,0)$, but asymptotic expansions are unknown and remain an open problem. The asymptotic behavior of variant $2 \mathrm{c}-2$ at $(0,0)$ is an open problem. Key for the last three columns: $\mathrm{R}=$ regular; $\mathrm{O}=$ osculatory; $\mathrm{IC}=$ infinite curvature; unbd $=$ unbounded; open $=$ open problem .

Proposition 2.1. For any fixed $p$ with $1 \leq p<\infty$, the solutions of MP 1, MPs $2 a$ and MPs $2 c-1$ and $2 c-3$ are in the $L_{p}(\Omega)$ function space if and only if the following integral is finite for any $\epsilon$ in the interval $(0,1]$ :

$$
\int_{0}^{\epsilon} \frac{1}{\left(f_{1}(x)-f_{2}(x)\right)^{p-1}} \mathrm{~d} x .
$$

Proof. We first note that, for the case of MP 1 and MP 2a, the comparison principle (see [Finn 1986]) gives that $u>0$. Also recall that there exist positive constants $M^{+}, M^{-}$, and $x_{o}$ such that

$$
\frac{M^{-}}{f_{1}(x)-f_{2}(x)}<u<\frac{M^{+}}{f_{1}(x)-f_{2}(x)} \text { for } 0<x<x_{o} .
$$

We now bound the integral $\int_{\Omega}|u|^{p} \mathrm{~d} A$ from above:

$$
\begin{aligned}
\int_{\Omega}|u|^{p} \mathrm{~d} A & =\int_{\Omega} u^{p} \mathrm{~d} A \quad(\text { since } u>0) \\
& =\int_{x=0}^{1} \int_{f_{2}(x)}^{f_{1}(x)} u^{p} \mathrm{~d} y \mathrm{~d} x=\int_{x=0}^{x_{o}} \int_{f_{2}(x)}^{f_{1}(x)} u^{p} \mathrm{~d} y \mathrm{~d} x+\int_{x=x_{o}}^{1} \int_{f_{2}(x)}^{f_{1}(x)} u^{p} \mathrm{~d} y \mathrm{~d} x \\
& \leq \int_{x=0}^{x_{o}} \int_{f_{2}(x)}^{f_{1}(x)} u^{p} \mathrm{~d} y \mathrm{~d} x+\int_{x=x_{o}}^{1} \int_{f_{2}(x)}^{f_{1}(x)} \max _{x_{o}<x<1} u^{p} \mathrm{~d} y \mathrm{~d} x
\end{aligned}
$$




$$
<\int_{x=0}^{x_{o}} \int_{f_{2}(x)}^{f_{1}(x)} \frac{\left(M^{+}\right)^{p}}{\left(f_{1}(x)-f_{2}(x)\right)^{p}} \mathrm{~d} y \mathrm{~d} x+\max _{x_{o}<x<1}(u)^{p} \int_{x=x_{o}}^{1} \int_{f_{2}(x)}^{f_{1}(x)} 1 \mathrm{~d} y \mathrm{~d} x .
$$

This last sum can also be written as

$$
\left(M^{+}\right)^{p} \int_{0}^{x_{o}} \frac{1}{\left(f_{1}(x)-f_{2}(x)\right)^{p-1}} \mathrm{~d} x+\max _{x_{o}<x<1}(u)^{p} \int_{x=x_{o}}^{1}\left(f_{1}(x)-f_{2}(x)\right) \mathrm{d} x .
$$

If $p$ is chosen so that the integral (18) is finite for any $\epsilon \in(0,1]$, then the first term of (19) is finite. Also, noting that $u$ is bounded away from the origin $\left(u \in C^{1}(\bar{\Omega} \backslash\{0\})\right)$ and that the domains $\Omega$ for the model problems are bounded domains, the second term of (19) is also finite. Thus if $p$ is chosen so that integral (18) is finite then the solution of MPs 1 and $2 \mathrm{a}$ are in the $L_{p}(\Omega)$ function space. We now bound the integral $\int_{\Omega}|u|^{p} \mathrm{~d} A$ from below:

$$
\begin{aligned}
\int_{\Omega}|u|^{p} \mathrm{~d} A & =\int_{x=0}^{x_{o}} \int_{f_{2}(x)}^{f_{1}(x)} u^{p} \mathrm{~d} y \mathrm{~d} x+\int_{x=x_{o}}^{1} \int_{f_{2}(x)}^{f_{1}(x)} u^{p} \mathrm{~d} y \mathrm{~d} x \\
& >\int_{x=0}^{x_{o}} \int_{f_{2}(x)}^{f_{1}(x)} u^{p} \mathrm{~d} y \mathrm{~d} x>\int_{x=0}^{x_{o}} \int_{f_{2}(x)}^{f_{1}(x)} \frac{\left(M^{-}\right)^{p}}{\left(f_{1}(x)-f_{2}(x)\right)^{p}} \mathrm{~d} y \mathrm{~d} x \\
& =\left(M^{-}\right)^{p} \int_{x=0}^{x_{o}} \frac{1}{\left(f_{1}(x)-f_{2}(x)\right)^{p-1}} \mathrm{~d} x .
\end{aligned}
$$

This gives that if $p$ is chosen so that integral (18) is not finite, then the solutions of MPs 1 and 2a are not in the $L_{p}$ function space.

The proof for MPs 2c-1 and 2c-3 is slightly more complicated because $u>0$ does not hold. A sketch of the proof for these cases is as follows. Since $u \in C^{1}(\bar{\Omega} \backslash\{0\})$, there is a neighborhood $\Omega_{s}$ of the singularity where the solution is either positive or negative. Using the approach above, it can be shown that $u \in L_{p}\left(\Omega_{s}\right)$ if and only if integral (18) is finite, which is equivalent to $u \in L_{p}(\Omega)$ since $u$ is bounded away from the singularity.

Corollary 2.1. (A) The solution of $M P 1$ is in the $L_{2-\delta}$ function space where $\delta>0$.

(B) The solution is in the $L_{1+1 / 2-\delta}$ function space for $M P 2 a-1$, is in the $L_{1+1 / 3-\delta}$ function space for $M P 2 a-2$, and is in the $L_{1+1 / 4-\delta}$ function space for $M P 2 a-3$, where $\delta>0$.

(C) The solution is in the $L_{1+2 / 3-\delta}$ function space for MPs $2 c-1$ and $2 c-3$, where $\delta>0$.

Note finally that all solutions of the Laplace-Young equation in a bounded domain $\Omega$ are in $L_{1}$, which is consistent with the physical interpretation that the volume of the fluid under the capillary surface is finite. 
2.2. Asymptotic Laplace-Young equation. There are no closed-form solutions for the Laplace-Young equation in domains with a corner or a cusp, but closed-form solutions exist for the following simplification of the Laplace-Young PDE. These closed-form solutions will be used in Section 4 for convergence studies of the numerical methodology we propose in Section 3.

Assuming the slope of the solution of the Laplace-Young boundary value problem ((1)-(2)) is steep, i.e., $|\nabla u| \gg 1$, we can approximate the PDE and the boundary condition, by ignoring the 1 in the denominator of the differential operator $T(\cdot)$, and obtain the following boundary value problem:

$$
\begin{array}{ll}
\nabla \cdot \tilde{T}(u)=u & \text { in } \Omega, \\
\vec{v}_{1} \cdot \tilde{T}(u)=\cos \gamma & \text { on } \partial \Omega_{1}, \\
\vec{v}_{2} \cdot \tilde{T}(u)=\cos \gamma & \text { on } \partial \Omega_{2},
\end{array}
$$

where

$$
\tilde{T}(u)=\frac{\nabla u}{|\nabla u|} .
$$

This approximation is called the "steep slope approximation" [King et al. 1999] of the Laplace-Young boundary value problem, and unbounded closed-form solutions of this boundary value problem are known for two types of domains: the unbounded corner domain of Figure 2, left [King et al. 1999] and the circular cusp domains of Figure 4 [Aoki 2007]. Also, it has been shown that the exact solutions of this boundary value problem are good asymptotic approximations of the solutions of the original Laplace-Young equation on the same domains [Miersemann 1993; Aoki 2007]. We shall refer to this boundary value problem as the asymptotic LaplaceYoung boundary value problem. Note that this boundary value problem is a rare case of a nonlinear PDE with nonlinear boundary conditions for which one can find closed-form solutions in some nontrivial domains.

2.2.1. Closed-form solutions. Corner domain (Figure 2, left, $\gamma+\alpha<\pi / 2$ ): Let $u \in C^{2}(\Omega)$ be a solution of the boundary value problem (20)-(22) on the unbounded corner domain defined as in (3)-(5) with the boundary functions

$$
f_{1}(x)=x \tan \alpha, \quad f_{2}(x)=-x \tan \alpha .
$$

If $\gamma+\alpha<\pi / 2$, then $u$ is given as the following closed-form expression in terms of the polar coordinate variables $r$ and $\theta$ :

$$
u(r, \theta)=\frac{\cos \theta-\sqrt{k^{2}-\sin ^{2} \theta}}{k r},
$$

where $k=\sin \alpha / \cos \gamma$. This precise property of the asymptotic function in (8) was first observed in [King et al. 1999]. 

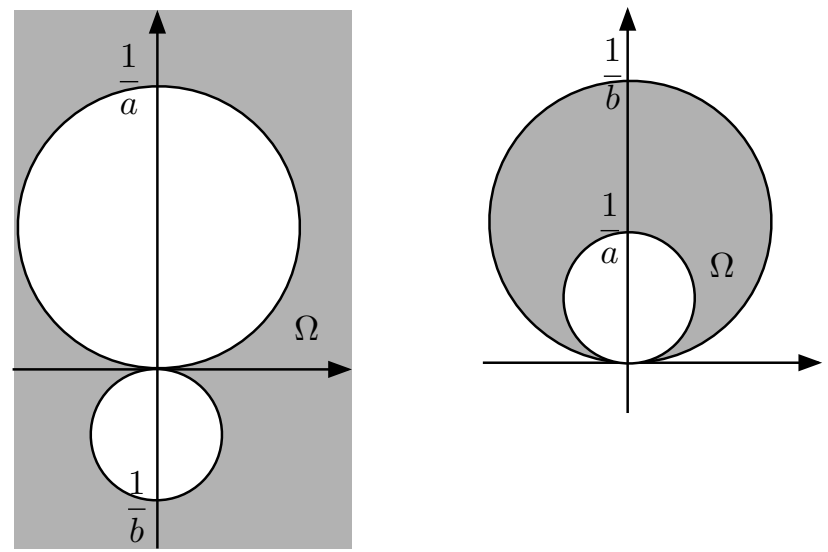

Figure 4. Circular cusp domains.

Circular cusp domain (Figure $4, \gamma \neq \pi / 2$ ): Let $u \in C^{2}(\Omega)$ be a solution of the boundary value problem (20)-(22) with $\gamma \neq \pi / 2$ and with the domain defined as

$$
\Omega:= \begin{cases}\left\{(x, y) \in \mathbb{R}^{2} \backslash\left(\bar{B}_{\frac{1}{2 a}}\left(0, \frac{1}{2 a}\right) \cup \bar{B}_{-\frac{1}{2 b}}\left(0, \frac{1}{2 b}\right)\right)\right\} & \text { for } b<0, \\ \left.(x, y) \in\left(B_{\frac{1}{2 b}}\left(0, \frac{1}{2 b}\right) \backslash \bar{B}_{\frac{1}{2 a}}\left(0, \frac{1}{2 a}\right)\right)\right\} & \text { for } b>0,\end{cases}
$$

where $B_{r}\left(x_{o}, y_{o}\right)$ is the open disc of radius $r$ centered at $\left(x_{o}, y_{o}\right)$, i.e.,

$$
B_{r}\left(x_{o}, y_{o}\right)=\left\{(x, y) \in \mathbb{R}^{2}:\left(x-x_{o}\right)^{2}+\left(y-y_{o}\right)^{2}<r^{2}\right\} .
$$

A closed-form expression for $u$ is given by

$$
u(p, q)=A p^{2}-2 \sqrt{1-A^{2}\left(q-q_{0}\right)^{2}} p-A\left(q-q_{0}\right)^{2}+A q_{0}^{2},
$$

where

$$
A=\frac{2 \cos \gamma}{a-b}, \quad q_{0}=\frac{a+b}{2},
$$

and $p$ and $q$ are the coordinate variables of the tangent cylindrical coordinate system introduced in [Moon and Spencer 1961], depicted in Figure 5 and defined as

$$
p=\frac{x}{x^{2}+y^{2}}, \quad q=\frac{y}{x^{2}+y^{2}} .
$$

This closed-form solution of the asymptotic Laplace-Young equation first appears in [Aoki 2007]. Note that $\lim _{(x, y) \rightarrow(0,0)} p=\infty$ and the solution (24) behaves like $1 / x^{2}$ as $x \rightarrow 0$, hence it exhibits a more severe singularity than the singularity of the asymptotic Laplace-Young PDE in a corner domain, which features a $1 / r$ singularity. 


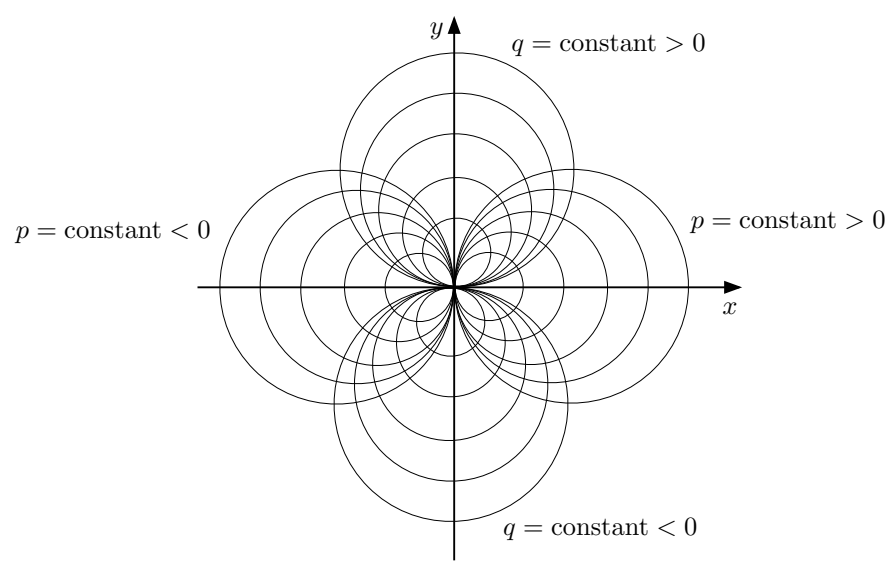

Figure 5. Tangent cylindrical coordinate system.

2.2.2. Model Problems 3 and 4. For the numerical experiments on the asymptotic Laplace-Young equation we consider the following model problems on the corner and cusp domains of Figure 3.

Let $u \in C^{2}(\Omega)$ be the function that satisfies the boundary value problem

$$
\begin{aligned}
\nabla \cdot \tilde{T}(u) & =u & & \text { in } \Omega, \\
\vec{v}_{1} \cdot \tilde{T}(u) & =\cos \gamma_{1} & & \text { on } \partial \Omega_{1}, \\
\vec{v}_{2} \cdot \tilde{T}(u) & =\cos \gamma_{2} & & \text { on } \partial \Omega_{2}, \\
u & =u_{\text {exact }} & & \text { on } \partial \Omega_{3} .
\end{aligned}
$$

Here with $\vec{v}_{1}, \vec{v}_{2}, \vec{v}_{3}$ are the exterior unit normal vectors on the boundaries $\partial \Omega_{1}$, $\partial \Omega_{2}, \partial \Omega_{3}$, while $\gamma_{1}, \gamma_{2}$ are the contact angles, and $u_{\text {exact }}$ is the closed-form solutions given in (23) or (24).

The bounded open domain $\Omega$ and boundaries $\partial \Omega_{1,2,3}$ are defined as in (16)-(17); see Figure 3. The boundary functions and the contact angles are chosen for each model problem as tabulated in Table 3, resulting in model problems with unbounded

\begin{tabular}{|ccccccc|}
\hline Name & $f_{1}(x)$ & $f_{2}(x)$ & $\gamma_{1}$ & $\gamma_{2}$ & & unbd? \\
MP 3 & $x \tan (\pi / 7)$ & $-x \tan (\pi / 7)$ & $\pi / 6$ & $\pi / 6$ & corner & yes \\
MP 4 & $-\sqrt{5^{2}-x^{2}}+5$ & $\sqrt{10^{2}-x^{2}}-10$ & $\pi / 6$ & $\pi / 6$ & circular cusp & yes \\
\hline
\end{tabular}

Table 3. Model Problems 3 and 4: asymptotic Laplace-Young equation in domains with a corner and a cusp. MP 3 has $\alpha=\pi / 7$ and $\gamma+\alpha<\pi / 2$, resulting in a solution that is unbounded at $(0,0)$. MP 4 has $\gamma \neq \pi / 2$, and its solution is also unbounded at $(0,0)$. 
solutions. The closed-form solutions of these two model problems are given by (23) and (24), respectively.

\section{Numerical method}

In this section, we propose a numerical methodology to accurately find global numerical approximations of singular solutions of the Laplace-Young equation in domains with a corner or a cusp. The starting point of our approach is the finite volume element method (FVEM) [Bank and Rose 1987; Aoki and De Sterck 2011] or the Galerkin finite element method (FEM) [Strang and Fix 1973; Brenner and Scott 1994], and two simple but crucial additional steps are made to arrive at a method that can capture the singular behavior. The first step is to consider a change of variable, with the new solution variable being smoother than the capillary height variable $u$ and more amenable to accurate numerical approximation. The second step is to solve the PDE numerically in a new coordinate system, which allows us to accurately represent the discontinuous behavior of the new solution variable at the singular point. We describe these two crucial ingredients of our methodology along with the FEM and FVEM discretizations, and show in the numerical results of Section 4 that this approach leads to a global approximation method for singular solutions of the Laplace-Young equation that recovers the proper asymptotic behavior, and is more accurate and has better convergence properties than numerical methods that were considered previously.

3.1. Change of variable. From the asymptotic analysis results (10)-(15) we observe that the solutions we wish to approximate have the asymptotic behavior

$$
\begin{array}{rlrl}
u(x, y) & =O\left(\frac{1}{f_{1}(x)-f_{2}(x)}\right) & & \text { as } x \rightarrow 0 \\
& =\frac{O(1)}{f_{1}(x)-f_{2}(x)} & \text { as } x \rightarrow 0 .
\end{array}
$$

This implies that, if we transform the unknown function $u(x, y)$ as follows, the new unknown function $v(x, y)$ is a bounded function:

$$
u(x, y)=\frac{v(x, y)}{f_{1}(x)-f_{2}(x)} .
$$

We aim to approximate the solution of the boundary value problem, $u(x, y)$, by numerically approximating the new unknown function $v(x, y)$. Since $v(x, y)$ is bounded while $u(x, y)$ is unbounded, we expect a better quality of numerical approximation.

3.2. Change of coordinates. An appropriate choice of coordinate system is essential for the asymptotic analysis of unbounded solutions of the Laplace-Young 
equation, as shown in [Miersemann 1993; Scholz 2004; Aoki 2007; Aoki and Siegel 2012]. We have observed that an appropriate choice of coordinate system is also beneficial for the numerical approximation of unbounded solutions.

For MP 1, we can observe as follows that the new unknown function $v$ is discontinuous at the origin. From (9), we know that the solution $u$ of MP 1 behaves like $\left(\cos \theta-\sqrt{k^{2}-\sin ^{2} \theta}\right) / k r$ near the origin $r=0$. This gives that the new unknown function $v$ behaves like $\left(\cos \theta-\sqrt{k^{2}-\sin ^{2} \theta}\right) / k$ near the origin. Hence, as $r \rightarrow 0, v$ approaches different values depending on the angle $\theta$, so the new unknown function $v$ has a jump discontinuity at the origin. Our idea is to expand the point of singularity on the boundary into a boundary line segment through a coordinate transformation in order to accurately approximate the discontinuous behavior of $v$.

For MP 2, since the boundaries for the cusp domain are curved boundaries, we would need special boundary elements (e.g., isoparametric elements) to accurately represent the cusp domain when approximating the unknown function through finite element approximation in the standard $(x, y)$ coordinate system. However, the change to $(s, t)$ coordinates introduced in [Aoki and Siegel 2012] and illustrated in Figure 6 transforms a cusp domain into a rectangular domain, and hence no special treatment is needed for curved boundaries.

We use this $(s, t)$ coordinate system for numerical simulation on domains with a corner or a cusp at $(0,0)$. The $(s, t)$ coordinate transformation as depicted in Figure 6 is given by

$$
t=\frac{2 y-\left(f_{1}+f_{2}\right)}{f_{1}-f_{2}}, \quad s=x .
$$

The Cartesian coordinates can be expressed using the above coordinate system as

$$
x=s, \quad y=\frac{t\left(f_{1}(s)-f_{2}(s)\right)+\left(f_{1}(s)+f_{2}(s)\right)}{2}=\frac{1+t}{2} f_{1}(s)+\frac{1-t}{2} f_{2}(s) .
$$

We have $y=f_{1}(x)$ when $t=1$ and $y=f_{2}(x)$ when $t=-1$, so the domain of interest in the curvilinear $(s, t)$ coordinate system can be written as (see Figure 6)

$$
\Omega=\left\{(s, t) \in \mathbb{R}^{2}: 0<s<1,-1<t<1\right\} .
$$

With some calculation, the left-hand side of the Laplace-Young PDE can be rewritten in the curvilinear coordinate system as

$$
\begin{aligned}
\nabla \cdot T(u)= & \frac{\partial}{\partial s} \frac{u_{x}}{\sqrt{1+u_{x}^{2}+u_{y}^{2}}}+\frac{f_{1}^{\prime}-f_{2}^{\prime}}{f_{1}-f_{2}} \frac{u_{x}}{\sqrt{1+u_{x}^{2}+u_{y}^{2}}} \\
& +\frac{\partial}{\partial t}\left(\frac{2}{f_{1}-f_{2}} \frac{u_{y}}{\sqrt{1+u_{x}^{2}+u_{y}^{2}}}+\left(-\frac{f_{1}^{\prime}+f_{2}^{\prime}}{f_{1}-f_{2}}-t \frac{f_{1}^{\prime}-f_{2}^{\prime}}{f_{1}-f_{2}}\right) \frac{u_{x}}{\sqrt{1+u_{x}^{2}+u_{y}^{2}}}\right),
\end{aligned}
$$



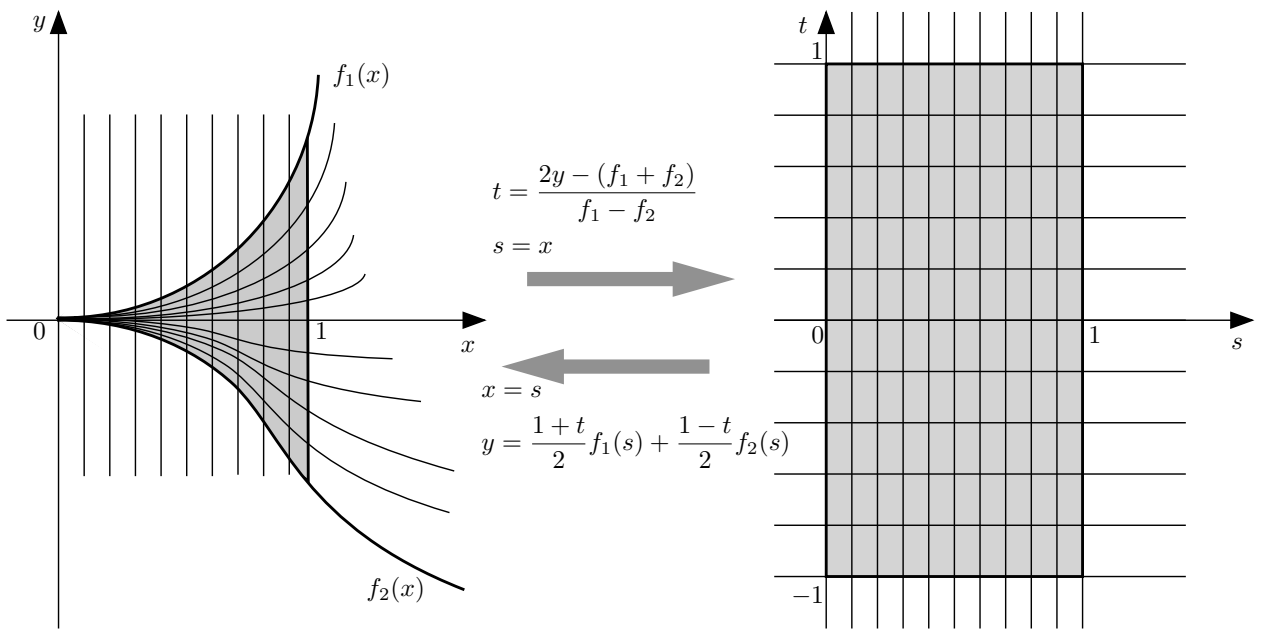

Figure 6. Coordinate transformation.

where

$$
u_{x}=\frac{v_{s}}{f_{1}-f_{2}}-\frac{v\left(f_{1}^{\prime}-f_{2}^{\prime}\right)}{\left(f_{1}-f_{2}\right)^{2}}-v_{t} \frac{\left(f_{1}^{\prime}+f_{2}^{\prime}\right)+t\left(f_{1}^{\prime}-f_{2}^{\prime}\right)}{\left(f_{1}-f_{2}\right)^{2}}, \quad u_{y}=\frac{2 v_{t}}{\left(f_{1}-f_{2}\right)^{2}} .
$$

The boundary conditions on $\partial \Omega_{1}$ and $\partial \Omega_{2}$ can be written as

$$
\begin{aligned}
\vec{v}_{1,2} \cdot T(u)= & \vec{v}_{1,2} \cdot \hat{s}\left(\frac{f_{1}-f_{2}}{2} \frac{u_{x}}{\sqrt{1+u_{x}^{2}+u_{y}^{2}}}\right) \\
& +\vec{v}_{1,2} \cdot \hat{t}\left(\frac{u_{y}}{\sqrt{1+u_{x}^{2}+u_{y}^{2}}}+\frac{-\left(f_{1}^{\prime}+f_{2}^{\prime}\right)-t\left(f_{1}^{\prime}-f_{2}^{\prime}\right)}{2} \frac{u_{x}}{\sqrt{1+u_{x}^{2}+u_{y}^{2}}}\right) \\
= & \sqrt{1+f_{1,2}^{\prime}(s)^{2}} \cos \gamma_{1,2} \quad \text { on } \partial \Omega_{1,2} .
\end{aligned}
$$

The boundary condition for boundary $\partial \Omega_{3}$ of MPs 1 and 2 is as in (26) but with zero on the right-hand side. The left-hand side and the boundary conditions of the asymptotic Laplace-Young PDE in the $(s, t)$ coordinate system can be obtained by just neglecting the 1 in the denominator in the expressions above. Note that the point $(x, y)=(0,0)$ corresponds to the line segment $(s=0, t \in[-1,1])$ in the $(s, t)$ coordinate system.

3.3. Discretized boundary value problem. In the numerical results of Section 4 we approximate the new unknown function $v(s, t)$ in the new coordinate variables $s$ and $t$ numerically on the Cartesian grid in $(s, t)$-space, as shown in Figure 7(a), and for comparison we also perform some calculations on the corner domain of Figure 7(b) without a change of coordinates. We now describe the Galerkin finite 


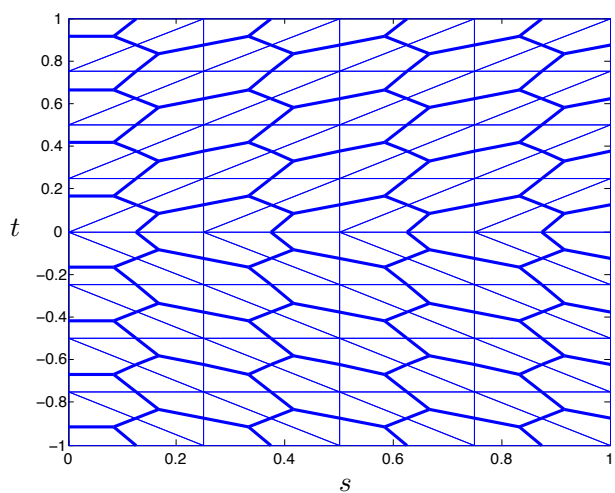

(a) $(s, t)$ space

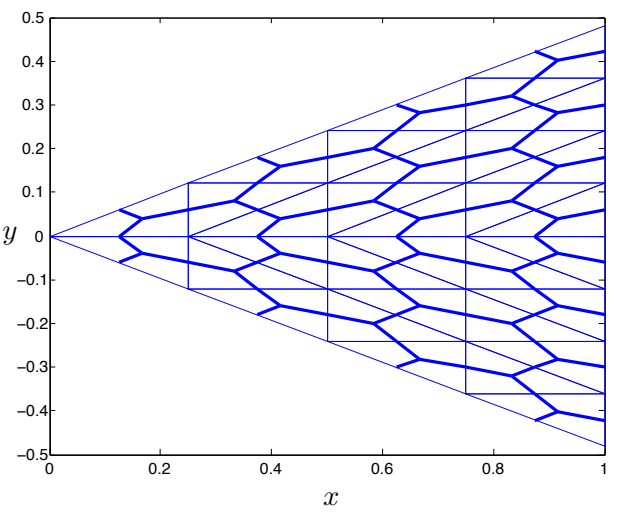

(b) a corner domain in $(x, y)$ space (without change of coordinates)

Figure 7. Finite elements and control volumes for the numerical methods. The thin lines give the finite element triangulation, which is used in both the FEM and the FVEM. The thick lines give the control volumes that are used in the FVEM. The grid in panel (a) can be used for corner domains or for cusp domains (depending on the boundary functions $f_{1}$ and $f_{2}$ that enter into the coordinate transformation formulas), and the grid in panel (b) is used for comparison simulations for corner domains (without coordinate transformation).

element method (FEM) and the finite volume element method (FVEM) discretizations.

3.3.1. Galerkin finite element method discretization. We follow the construction of the finite element space presented in Chapter 3 of Brenner and Scott [1994]. Let $N_{\text {node }}$ be the number of nodes created by finite element triangulation of the domain and $\mathcal{N}$ be the set of indices of the nodes, i.e., $\mathcal{N}=\left\{1,2, \ldots, N_{\text {node }}\right\}$. The triangulation of the domain is as depicted in Figure 7(a) (or Figure 7(b) for the corner problem without a change of coordinates). Also, we let $\mathcal{N}_{\text {Dirichlet }}$ be the indices of the nodes on the boundary with Dirichlet boundary condition. That is to say, for MPms 3 and 4,

$$
\left(s_{i}, t_{i}\right) \in \overline{\partial \Omega_{3}} \Rightarrow i \in \mathcal{N}_{\text {Dirichlet }},
$$

where $\left(s_{i}, t_{i}\right)$ is the location of the $i$-th node, and for MPs 1 and $2 \mathcal{N}_{\text {Dirichlet }}=\varnothing$ since there is no Dirichlet boundary. Let $\phi_{i}(s, t)$ be the standard continuous piecewise linear nodal basis function (tent function) that corresponds to node $i$ in the finite 
element triangulation on domain $\Omega$. We have

$$
\phi_{i}\left(s_{j}, t_{j}\right)=\delta_{i, j}
$$

where $\delta_{i, j}$ is the Kronecker delta function. We approximate the unknown function $v$ with a linear combination of these basis functions, i.e.,

$$
v \approx v^{h}:=\sum_{i=1}^{N_{\text {node }}} c_{i} \phi_{i}
$$

The $\left\{c_{1}, c_{2}, \ldots, c_{N_{\text {node }}}\right\}$ are the unknowns of the discretized boundary value problem. The Galerkin finite element discretization of MPs 1 and 2 can then be written as follows (the discretization of MPs 3 and 4 can be derived similarly):

$$
\int_{\Omega}\left(\nabla \cdot T\left(\frac{\sum_{i=1}^{N_{\text {node }}} c_{i} \phi_{i}}{f_{1}(s)-f_{2}(s)}\right)\right) \phi_{j} \mathrm{~d} A=\int_{\Omega} \frac{\sum_{i=1}^{N_{\text {node }}} c_{i} \phi_{i}}{f_{1}(s)-f_{2}(s)} \phi_{j} \mathrm{~d} A
$$

for $j \in \mathcal{N} \backslash \mathcal{N}_{\text {Dirichlet }}$,

$$
\frac{c_{i}}{f_{1}\left(s_{i}\right)-f_{2}\left(s_{i}\right)}=u_{\text {exact }}\left(s_{i}, t_{i}\right) \quad \text { for } i \in \mathcal{N}_{\text {Dirichlet }}
$$

By the divergence theorem we can rewrite (27) as

$$
\begin{gathered}
\int_{\partial \Omega}\left(v \cdot T\left(\frac{\sum_{i=1}^{N_{\text {node }}} c_{i} \phi_{i}}{f_{1}(s)-f_{2}(s)}\right)\right) \phi_{j} \mathrm{~d} l-\int_{\Omega}\left(T\left(\frac{\sum_{i=1}^{N_{\text {node }}} c_{i} \phi_{i}}{f_{1}(s)-f_{2}(s)}\right)\right) \cdot \nabla \phi_{j} \mathrm{~d} A \\
=\int_{\Omega} \sum_{i=1}^{N_{\text {node }}} c_{i} \frac{\phi_{i} \phi_{j}}{f_{1}(s)-f_{2}(s)} \mathrm{d} A \\
\quad \text { for } j \in \mathcal{N} \backslash \mathcal{N}_{\text {Dirichlet }} .
\end{gathered}
$$

By imposing the boundary conditions (26), we obtain the following system of equations:

$$
\begin{gathered}
\int_{\Omega}\left(T\left(\frac{\sum_{i=1}^{N_{\text {node }}} c_{i} \phi_{i}}{f_{1}(s)-f_{2}(s)}\right)\right) \cdot \nabla \phi_{j} \mathrm{~d} A-\sum_{i=1}^{N_{\text {node }}} c_{i} \int_{\Omega} \frac{\phi_{i} \phi_{j}}{f_{1}(s)-f_{2}(s)} \mathrm{d} A \\
\quad=\int_{\partial \Omega_{1}} \sqrt{1+f_{1}^{\prime}(s)^{2}} \cos \gamma_{1} \phi_{j} \mathrm{~d} l+\int_{\partial \Omega_{2}} \sqrt{1+f_{2}^{\prime}(s)^{2}} \cos \gamma_{2} \phi_{j} \mathrm{~d} l \\
\quad \text { for } j \in \mathcal{N} \backslash \mathcal{N}_{\text {Dirichlet }} .
\end{gathered}
$$

After some calculation we can rewrite (29) together with (28) as the following system of nonlinear equations: 


$$
\begin{aligned}
& \int_{t=-1}^{1} \int_{s=0}^{1}\left(\phi_{j}\right)_{s}\left(\frac{f_{1}-f_{2}}{2} \frac{u_{x}^{h}}{\sqrt{1+\left(u_{x}^{h}\right)^{2}+\left(u_{y}^{h}\right)^{2}}}\right) \\
& +\left(\phi_{j}\right)_{t}\left(\frac{u_{y}^{h}}{\sqrt{1+\left(u_{x}^{h}\right)^{2}+\left(u_{y}^{h}\right)^{2}}}+\frac{-\left(f_{1}^{\prime}+f_{2}^{\prime}\right)-t\left(f_{1}^{\prime}-f_{2}^{\prime}\right)}{2} \frac{u_{x}^{h}}{\sqrt{1+\left(u_{x}^{h}\right)^{2}+\left(u_{y}^{h}\right)^{2}}}\right) \mathrm{d} s \mathrm{~d} t \\
& -\sum_{i=1}^{N_{\text {node }}} c_{i} \int_{t=-1}^{1} \int_{s=0}^{1} \phi_{i} \phi_{j} \mathrm{~d} s \mathrm{~d} t \\
& =\int_{\partial \Omega_{1}} \sqrt{1+f_{1}^{\prime}(s)^{2}} \cos \gamma_{1} \phi_{j} \mathrm{~d} l+\int_{\partial \Omega_{2}} \sqrt{1+f_{2}^{\prime}(s)^{2}} \cos \gamma_{2} \phi_{j} \mathrm{~d} l
\end{aligned}
$$

for $j \in \mathcal{N} \backslash \mathcal{N}_{\text {Dirichlet }}$,

$$
c_{i}=u_{\text {exact }}\left(x_{i}, y_{i}\right) \quad \text { for } i \in \mathcal{N}_{\text {Dirichlet }},
$$

where

$$
\begin{aligned}
& u_{x}^{h}=\sum_{i=1}^{N_{\text {node }}} c_{i}\left(\frac{\left(\phi_{i}\right)_{s}}{f_{1}-f_{2}}-\frac{\left(\phi_{i}\right)\left(f_{1}^{\prime}-f_{2}^{\prime}\right)}{\left(f_{1}-f_{2}\right)^{2}}-\left(\phi_{i}\right)_{t} \frac{\left(f_{1}^{\prime}+f_{2}^{\prime}\right)+t\left(f_{1}^{\prime}-f_{2}^{\prime}\right)}{\left(f_{1}-f_{2}\right)^{2}}\right) \\
& u_{y}^{h}=\sum_{i=1}^{N_{\text {node }}} c_{i} \frac{2\left(\phi_{i}\right)_{t}}{\left(f_{1}-f_{2}\right)^{2}}
\end{aligned}
$$

and $\left(\phi_{i}\right)_{s}$ and $\left(\phi_{i}\right)_{t}$ are the partial derivatives of $\phi_{i}$ with respect to $s$ and $t$. We can construct a system of nonlinear equations by integrating each of the terms in (30) numerically. Note that although we are integrating the unbounded functions $v^{h} \phi_{j} /\left(f_{1}(s)-f_{2}(s)\right)$, due to the change of coordinates the area element $\mathrm{d} A$ becomes $\left(f_{1}(s)-f_{2}(s)\right) / 2 \mathrm{~d} s \mathrm{~d} t$, hence the integrand becomes $2 v^{h} \phi_{j}$, a piecewise quadratic polynomial; hence we avoid singular integration. We solve this system of nonlinear equations with the Levenberg-Marquardt method to obtain the unknowns $\left\{c_{1}, c_{2}, \ldots, c_{N_{\text {node }}}\right\}$. This gives a numerical approximation for $v$, and hence a numerical approximation of the solution of the boundary value problem $u$.

3.3.2. Finite volume element method discretization. The finite volume element method (FVEM) is a type of Petrov-Galerkin method that uses piecewise constant functions as test functions in the weak form, instead of using the finite element basis functions as in the Galerkin FEM. The test functions for the FVEM are chosen as follows:

$$
\psi_{j}(s, t)= \begin{cases}1 & \text { if }(s, t) \in \Omega_{j} \\ 0 & \text { otherwise }\end{cases}
$$

where the $\Omega_{j}$ are control volumes constructed as in [Bank and Rose 1987] (note that in [Bank and Rose 1987] the control volumes are called "boxes"). As depicted in Figure 7(a) (and Figure 7(b)), the control volumes $\left\{\Omega_{j}\right\}_{j=1}^{N_{\text {node }}}$ are constructed by 
first computing the centroids of the finite element triangles, and then connecting those element centroids with the midpoints of the finite element triangle edges. This construction divides each finite element triangle into three quadrilaterals. The control volume $\Omega_{j}$ for finite element node $j$ is then constructed as the union of the quadrilaterals adjacent to node $j$.

By substituting the test functions $\phi_{j}$ by $\psi_{j}$ in the Galerkin finite element discretization (27) and after some calculation, we obtain the following system of nonlinear equations for the FVEM, where $u_{x}$ and $u_{y}$ are defined as in (31) and (32):

$$
\begin{aligned}
& \int_{\partial \Omega_{j}} \vec{v} \cdot \hat{s}\left(\frac{f_{1}-f_{2}}{2} \frac{u_{x}^{h}}{\sqrt{1+\left(u_{x}^{h}\right)^{2}+\left(u_{y}^{h}\right)^{2}}}\right) \\
& +\vec{v} \cdot \hat{t}\left(\frac{u_{y}^{h}}{\sqrt{1+\left(u_{x}^{h}\right)^{2}+\left(u_{y}^{h}\right)^{2}}}+\frac{-\left(f_{1}^{\prime}+f_{2}^{\prime}\right)-t\left(f_{1}^{\prime}-f_{2}^{\prime}\right)}{2} \frac{u_{x}^{h}}{\sqrt{1+\left(u_{x}^{h}\right)^{2}+\left(u_{y}^{h}\right)^{2}}}\right) \mathrm{d} l \\
& \quad=\int_{\partial \Omega_{1} \cap \partial \Omega_{j}} \sqrt{1+f_{1}^{\prime}(s)^{2}} \cos \gamma_{1} \mathrm{~d} l+\int_{\partial \Omega_{2} \cap \partial \Omega_{j}} \sqrt{1+f_{2}^{\prime}(s)^{2}} \cos \gamma_{2} \mathrm{~d} l \\
& \text { for } j \in \mathcal{N} \backslash \int_{\Omega_{\text {Dirichlet }}} \phi_{i} \mathrm{~d} s \mathrm{~d} t
\end{aligned}
$$

Again, we avoid singular integration by the change of coordinates, hence the integration can be done numerically without any special treatment for singular integration. We solve the resulting system of nonlinear equations using the LevenbergMarquardt method.

Note that we choose the triangulations of Figures 7(a) and 7(b) symmetric with respect to the $t=0$ and $y=0$ axes, respectively. While this is not a requirement, we made this choice because some of our model problems are symmetric with respect to the $t=0$ and $y=0$ axes, and this choice of grid leads to numerical solutions that closely retain this symmetry.

The FEM is known to achieve optimality in the energy norm for linear elliptic PDEs, but it does not have a local conservation property. The FVEM has a local conservation property like the finite volume method; however, it does not necessarily produce an optimal approximation. We have conducted numerical experiments using both methods, and the results we obtained were very similar. For brevity, we mainly present the numerical experiment results obtained by the FVEM in this paper, except in a few places where we compare them with the Galerkin FEM.

\section{Numerical results}

We now show that the numerical approximations we obtain with the computational methodology proposed in Section 3 for singular solutions of the Laplace-Young 


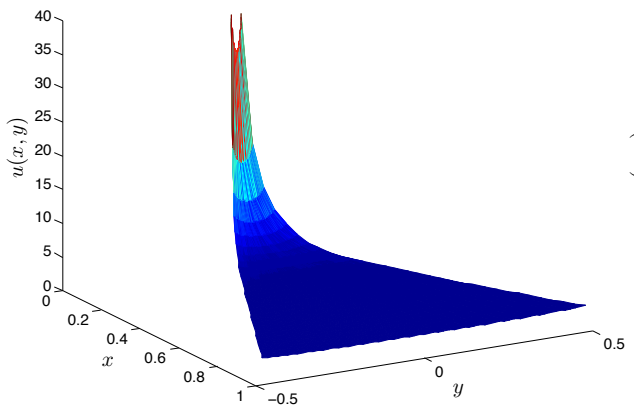

(a) MP 1-1: corner problem

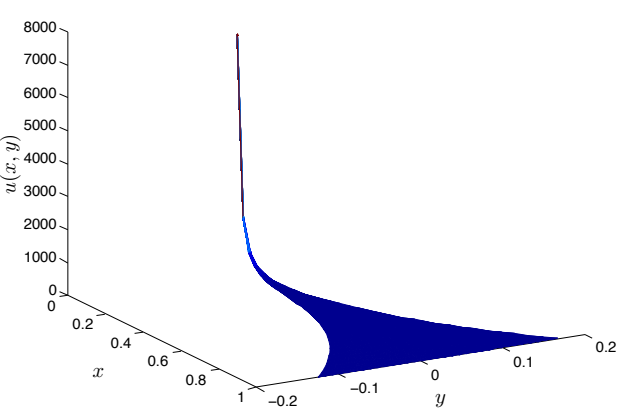

(b) MP 2a-1: cusp problem

Figure 8. MPs 1-1 and 2a-1. FVEM solution on the $(s, t)$-type grid of Figure 7 (a) with $33 \times 65$ nodes. Surface plots of the unbounded capillary surfaces in the corner and cusp domains.

equation in domains with a corner or a cusp are accurate global approximations. As an initial illustration, surface plots for two numerical approximations of singular solutions of the Laplace-Young equation in domains with a corner and with a cusp are shown in Figure 8. In what follows, we first show how our numerical methods obtain accurate global solutions for unbounded solutions of the Laplace-Young equation in domains with a corner or a cusp, by comparing with known asymptotic expansions and formal asymptotic series. We then numerically investigate the convergence behavior of the methods we propose using known closed-form unbounded solutions for the asymptotic Laplace-Young equation. The numerical results confirm that the computational methods we propose are accurate and have good convergence properties, and that they can be used with confidence to numerically investigate open problems on asymptotic solutions of the Laplace-Young equation in Section 5.

4.1. Laplace-Young equation: asymptotic behavior. We now investigate how well our numerical solutions can approximate the singular behavior by comparing the numerical solutions to known asymptotic solutions for the Laplace-Young equation.

MP 1: corner problem. As given in (8), the leading-order term of the asymptotic series solution of the Laplace-Young equation at a sharp corner is known. In Figure 9, we plot a horizontal cross-section (a cross-section along the $x$-axis or $s$ axis; see Figure 6) of the numerical approximation and the asymptotic approximation in log-log scale. In Figure 10, we plot a vertical cross-section (a cross-section along the line $x=1 / 2^{5}$ or $s=1 / 2^{5}$; see Figure 6 ) of the numerical approximation and the asymptotic approximation.

In order to illustrate the crucial benefits of the change of variable and change of coordinates that are the essential building blocks of the numerical methodology 
we proposed in Section 3, we compare four different choices for obtaining the numerical approximation using the FVEM: with or without change of variable, and with or without change of coordinates. The only published work on numerical approximation of singular capillary surfaces [Scott et al. 2005] also uses the FVEM, but it does not use a change of variable nor a change of coordinates, and thus corresponds to Figures 9 and 10.

As can be seen in Figures 9 and 10, the change of variable and the change of coordinates proposed in Sections 3.1 and 3.2 are very beneficial for the accuracy of the numerical approximations on a domain with a sharp corner near the singularity. Note that we cannot conduct a numerical convergence study for these unbounded solutions of the Laplace-Young equation, as there is no known closed-form solution.

MP 2: cusp problem. We now consider the Laplace-Young equation in a domain with a cusp. Unbounded cusp solutions are known to have a more severe singularity than the sharp corner problem. The leading-order term of the asymptotic series solution is known; see (11). Also, as shown in Lemma 2.3 of [Aoki and Siegel 2012], the first two terms of the formal asymptotic series $\tilde{u}$ are known:

$$
\tilde{u}=\frac{\cos \gamma_{1}+\cos \gamma_{2}}{f_{1}(s)-f_{2}(s)}-\sqrt{1-\left(\frac{\cos \gamma_{1}(t+1)+\cos \gamma_{2}(t-1)}{2}\right)^{2}} \frac{f_{1}^{\prime}(s)-f_{1}^{\prime}(s)}{f_{1}(s)-f_{2}(s)} .
$$

The formal asymptotic series of a boundary value problem is a series that satisfies the PDE and the boundary condition asymptotically, but, as opposed to the case of an asymptotic expansion, a bound on the error has not been proven. (There is no $O(\cdot)$ term in (34), but there is one in the asymptotic expansion (11).)

As can be seen in Figure 11, the numerical solution we obtain for MP 2 with the change of variable and the change of coordinates proposed in Sections 3.1 and 3.2 accurately approximates the singular behavior.

Although it is not known if the second-order term of the formal asymptotic series of this problem is in fact the second-order term of the asymptotic series solution, it can be seen in Figure 11 that the numerical solution appears to match better with the second-order formal asymptotic series than with the first-order asymptotic solution. It is particularly interesting that the domain where the asymptotic approximation is a good approximation seems to expand by adding a second term to the asymptotic series.

\subsection{Asymptotic Laplace-Young equation: convergence study. In the previous} section, we have shown that the numerical approximations with a change of variable and a change of coordinates as proposed in Sections 3.1 and 3.2 exhibit the correct singular behavior for singular solutions of the Laplace-Young equation. Since our interest is to obtain global approximations which are accurate both at the singularity and away from the singularity, we now show that the numerical solution in fact 


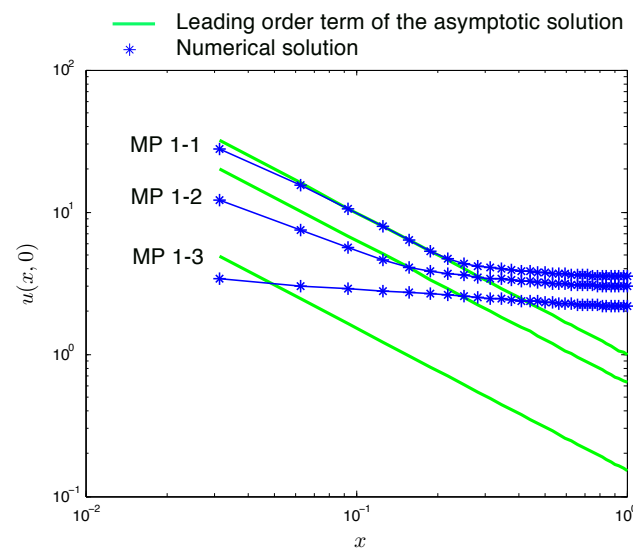

(a) without change of coordinates and without change of variable

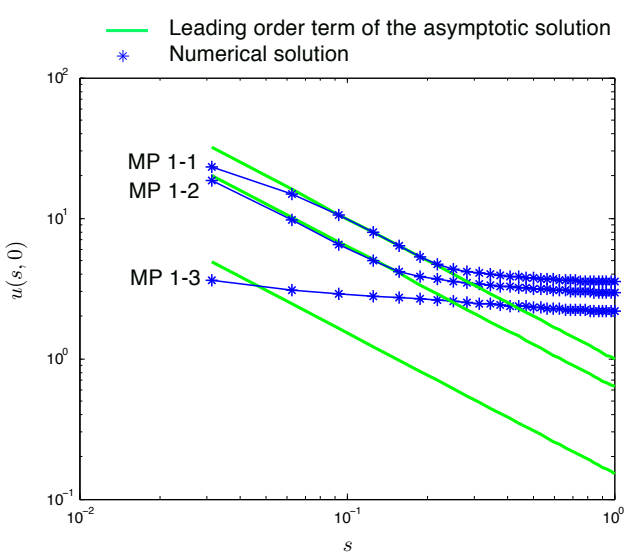

(c) with change of coordinates and without change of variable

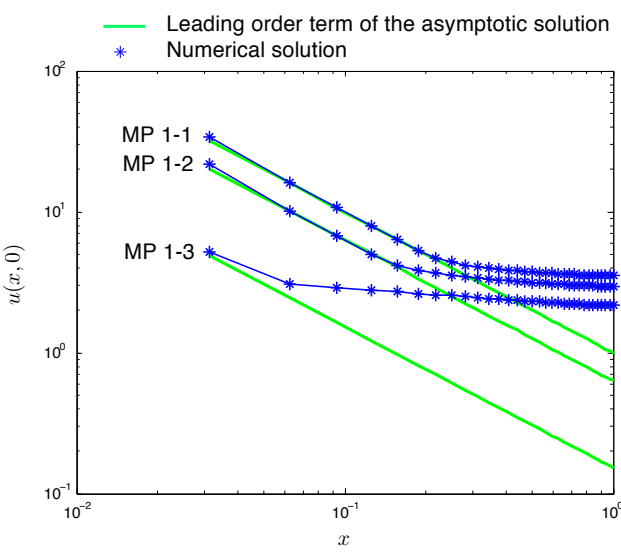

(b) without change of coordinates and with change of variable

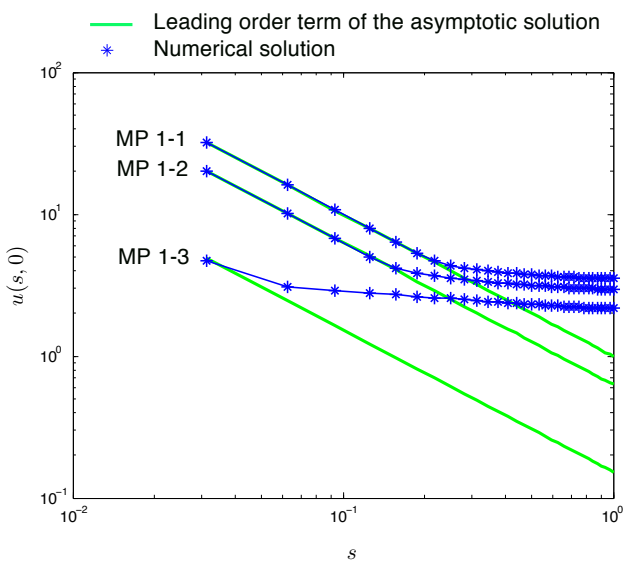

(d) with change of coordinates and with change of variable

Figure 9. MPs 1-1, 1-2 and 1-3 (unbounded corner solutions). Panels (a) and (b) show FVEM solutions on the $(x, y)$-type grid of Figure 7(b) with 1089 nodes (no change of coordinates). Panels (c) and (d) show FVEM solutions on the $(s, t)$-type grid of Figure 7 (a) with $33 \times 65$ nodes (with change of coordinates). Panels (a) and (c) are for computation of the original variable $u$, and panels (b) and (d) are for computation of the transformed variable $v$. The log-log plots show a comparison of the numerical solutions and the first-order asymptotic approximations in a horizontal cross section at $y=0$ or $t=0$. Panel (d) clearly gives the most accurate numerical solutions. 


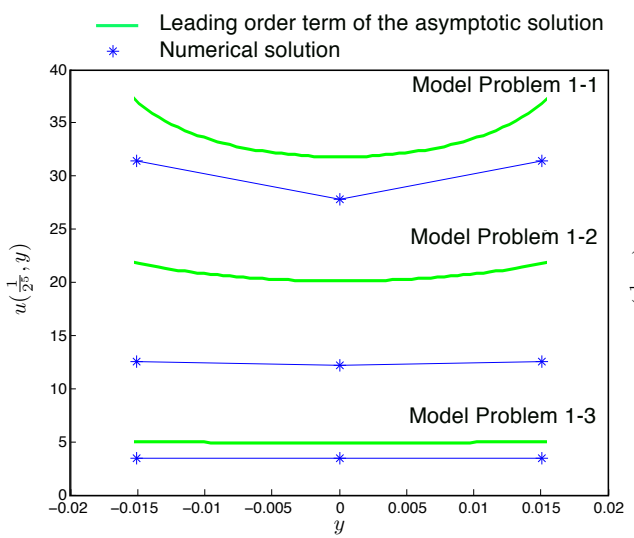

(a) without change of coordinates and without change of variable

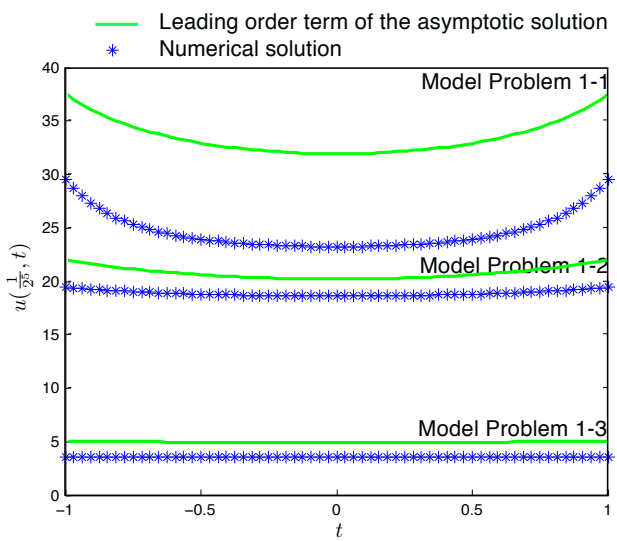

(c) with change of coordinates and without change of variable

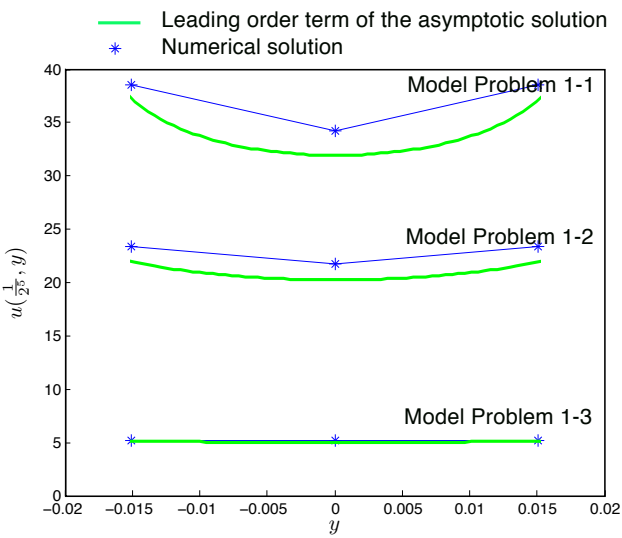

(b) without change of coordinates and with change of variable

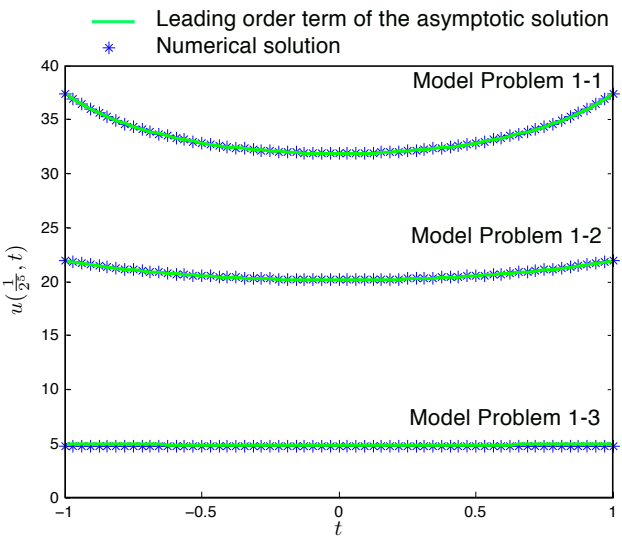

(d) with change of coordinates and with change of variable

Figure 10. MPs 1-1, 1-2, and 1-3 (unbounded corner solutions). Panels (a) and (b) show FVEM solutions on the $(x, y)$-type grid of Figure 7(b) with 1089 nodes (no change of coordinates). Panels (c) and (d) show FVEM solutions on the $(s, t)$-type grid of Figure 7 (a) with $33 \times 65$ nodes (with change of coordinates). Panels (a) and (c) are for computation of the original variable $u$, and panels (b) and (d) are for computation of the transformed variable $v$. The plots show a comparison of the numerical solutions and the first-order asymptotic approximations in a vertical cross section at $x=1 / 2^{5}$ or $s=1 / 2^{5}$ (the grid points closest to the singular point). Panel (d) clearly gives the most accurate numerical solutions. 

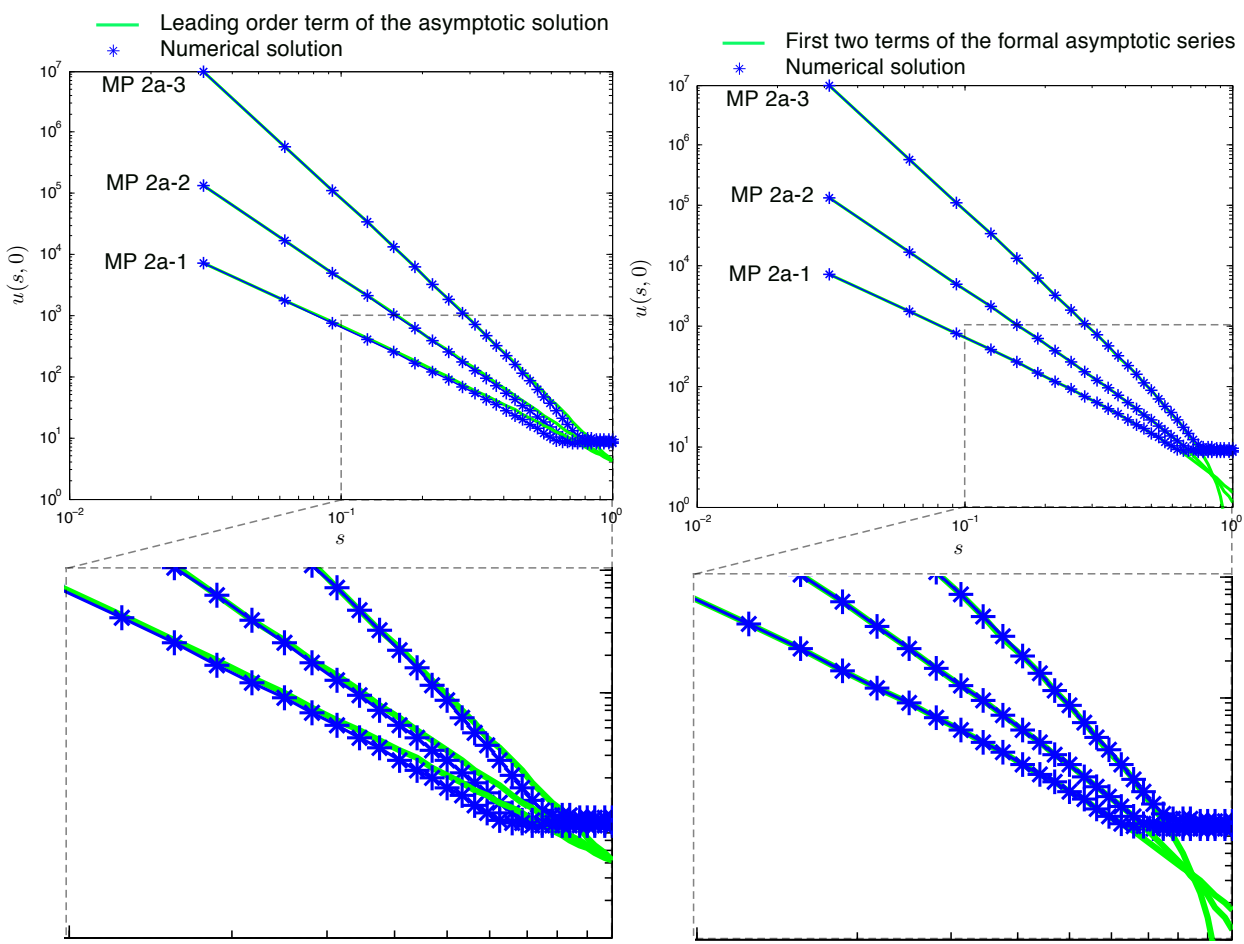

Figure 11. MPs 2a-1, 2a-2 and 2a-3 (unbounded cusp solutions). FVEM solutions on the $(s, t)$-type grid of Figure 7(a) with $33 \times 65$ nodes (with change of coordinates and with change of variable). The log-log plots in the left panels show a comparison of the numerical solutions with the first-order asymptotic solution in a horizontal cross section at $t=0$. The log-log plots in the right panels show a comparison of the numerical solutions with the first two terms of the formal asymptotic series in a horizontal crosssection at $t=0$. It is clear that accurate numerical solutions are obtained.

converges to the exact solution everywhere. It would be desirable to conduct a numerical convergence study for the Laplace-Young equation, but there is no known closed-form singular solution, and hence we cannot conduct a numerical convergence study. As we have discussed in Section 2.2, there are known exact solutions of the asymptotic Laplace-Young equation, and it is known that they have the same singular behavior as the corresponding solutions of the Laplace-Young equation. We therefore conduct a numerical convergence study for the asymptotic LaplaceYoung equation in corner and cusp domains. Since the exact solution is in the $L_{1}$ function space but not in $L_{2}$, we conduct the convergence study in the $L_{1}$ norm. 


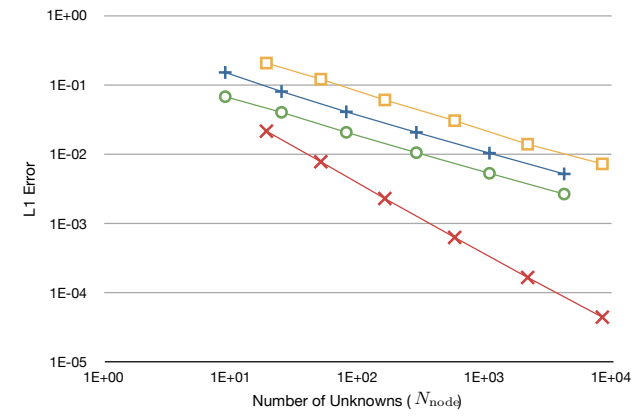

+ without change of coordinates and without change of variable ㅁ. with change of coordinates and without change of variable

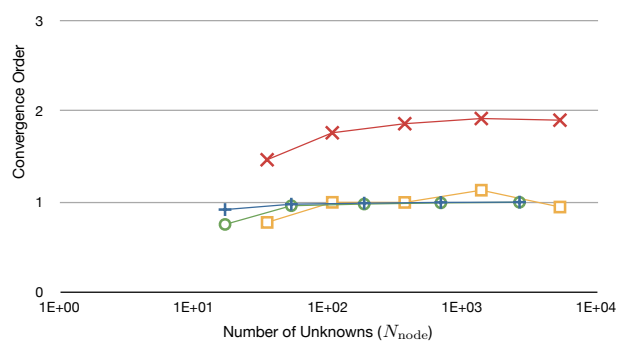

- without change of coordinates and with change of variable * with change of coordinates and with change of variable

Figure 12. MP 3 (unbounded corner solution for asymptotic Laplace-Young). FVEM solutions on $(s, t)$-type grids (Figure 7(a)) and on $(x, y)$-type grids (Figure 7(b)), with and without change of variable. The plots show $L_{1}$ convergence of the numerical solutions obtained by the FVEM to the closed-form solution. The plots indicate that all four approaches converge, but it is clear that the method with change of variable and with change of coordinates converges significantly faster (with nearly second-order accuracy) than the other approaches.

MP 3: corner problem. As can be seen in Figure 12, the FVEM numerical approximation with change of variable and change of coordinates as proposed in Sections 3.1 and 3.2 converges to the closed-form solution nearly quadratically, whereas the other approaches (no change of variable or no change of coordinates) only converge linearly.

MP 4: cusp problem. We have also conducted a numerical convergence study for the circular cusp problem, where the solution has a more severe singularity than for the corner problem. For this problem, we have used both the Galerkin finite element method (FEM) and the finite volume element method (FVEM) to show that both numerical schemes work well with the change of variable and the change of coordinates proposed in Sections 3.1 and 3.2. As can be seen in Figure 13, both the FEM and the FVEM achieve near-quadratic convergence with the change of variable and change of coordinates, while only linear convergence can be achieved without change of variable.

\section{Conjectures on open problems}

As shown in the previous section, we can obtain a globally accurate approximation of unbounded solutions of the Laplace-Young equation using the numerical 


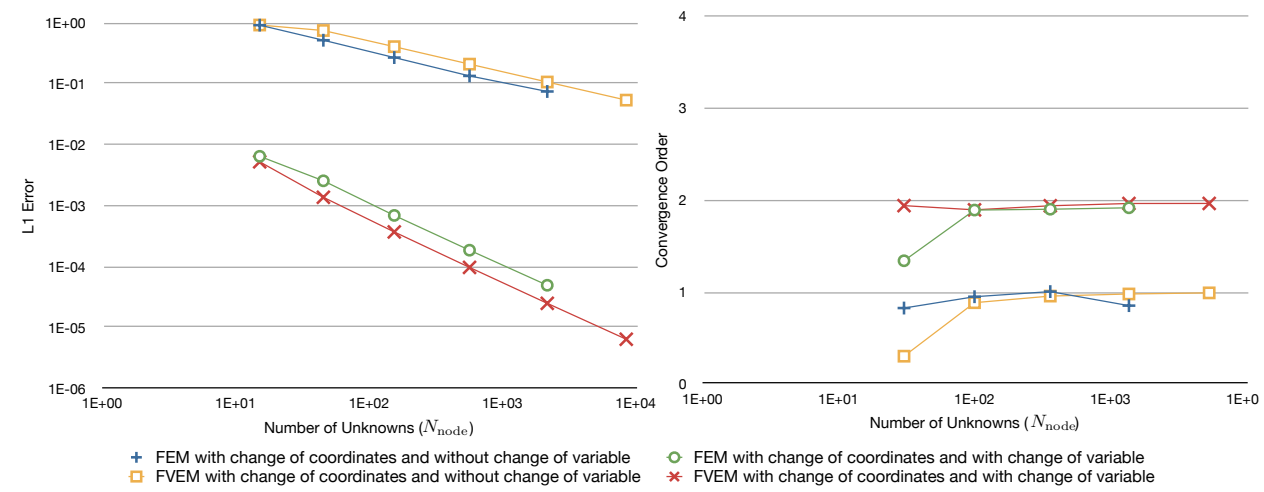

Figure 13. MP 4 (unbounded cusp solution for asymptotic Laplace-Young). FVEM and FEM solutions on $(s, t)$-type grids (Figure 7(a), with change of coordinates), with and without change of variable. The plots show $L_{1}$ convergence of the numerical solutions obtained by the FVEM and FEM to the closed-form solution. The plots indicate that all four approaches converge, but it is clear that the methods with change of variable converge significantly faster (with nearly second-order accuracy).

methodology proposed in Section 3. We now numerically approximate the solutions of two problems where the singular behavior is not known yet analytically. Our numerical results will allow us to formulate conjectures on asymptotic behavior for these open problems, which may guide further analytical study of these open problems.

\subsection{Open problem 1: osculatory cusp with nonsupplementary contact angles.} As stated in Section 2.1.2, the leading-order asymptotic behavior of the unbounded solution of the Laplace-Young equation at an osculatory cusp is not known: In summary, a proof for the leading-order asymptotic behavior could not be obtained in [Aoki and Siegel 2012] for the osculatory cusp because the authors were not able to determine the formal asymptotic series. As shown in Lemma 2.2 of that paper, the first two terms of the formal asymptotic series are known for the osculatory cusp case up to an additive constant in the coefficient of the second-order term, i.e.,

$$
\begin{aligned}
& \tilde{u}=\frac{\cos \gamma_{1}+\cos \gamma_{2}}{f_{1}(s)-f_{2}(s)} \\
& \quad+\left(-\sqrt{1-\left(\frac{\cos \gamma_{1}(t+1)+\cos \gamma_{2}(t-1)}{2}\right)^{2}}+C_{1}\right) \frac{f_{1}^{\prime}(s)-f_{1}^{\prime}(s)}{f_{1}(s)-f_{2}(s)},
\end{aligned}
$$

where $\tilde{u}$ asymptotically satisfies the boundary value problem, $C_{1}=0$ if the cusp is not an osculatory cusp, and $C_{1}$ is unknown if it is an osculatory cusp. One can see 
from the proofs in [Aoki and Siegel 2012] that the unknown additive constant $C_{1}$ is the elusive key to the proof of the leading-order behavior of the osculatory cusp problem. The coefficient $C_{1}$ is unknown and may depend on the specific functional form of the boundary functions $f_{1}(s)$ and $f_{2}(s)$.

Physical intuition suggests that the singular behavior of the unbounded capillary surface near a sharp corner or a cusp may be governed only by the distance between the two boundaries forming the sharp corner or cusp. In other words, one may think that the asymptotic behavior should only depend on $f_{1}(s)-f_{2}(s)$ and its derivatives and not on $f_{1}(s)$ and $f_{2}(s)$ separately. This would imply that the formal asymptotic series would be the same for the four MPs $2 \mathrm{a}-2$ and $2 \mathrm{~b}$, since $f_{1}(s)-f_{2}(s)=7 / 24 x^{3}$ for all these cases. If so, then $C_{1}=0$ is required also for the osculatory cusps of MPs $2 \mathrm{~b}$, since $C_{1}=0$ for the regular cusp of MP 2a-2. But it is also possible that $C_{1}$ depends on the precise functional form of $f_{1}(s)$ and $f_{2}(s)$.

In order to investigate this, we now numerically approximate the second-order term of the formal asymptotic series by the following change of variable for the unknown function $u$ :

$$
u(s, t)=\frac{\cos \gamma_{1}+\cos \gamma_{2}}{f_{1}(s)-f_{2}(t)}+w(s, t) \frac{f_{1}^{\prime}(s)-f_{2}^{\prime}(s)}{f_{1}(s)-f_{2}(s)} .
$$

We numerically approximate the new unknown function $w(s, t)$ in $(s, t)$ coordinates, and we plot the second-order term $w(s, t)\left(f_{1}^{\prime}(s)-f_{2}^{\prime}(s)\right) /\left(f_{1}(s)-f_{2}(s)\right)$ (or equivalently, $\left.u(s, t)-\left(\cos \gamma_{1}+\cos \gamma_{2}\right) /\left(f_{1}(s)-f_{2}(t)\right)\right)$ obtained from the numerical approximation in Figure 14.

As can be seen in Figure 14, the known second-order term of the formal asymptotic series for the regular cusp (MP 2a-2) is approximated correctly using the change of variable (36). Also, Figure 14 shows that the second-order term of the formal asymptotic series of the osculatory cusp case differs from the regular cusp case and is shifted up by constants, consistent with (35). These numerical results guide us in conjecturing that the additive constant $C_{1}$ of the coefficient of the second-order formal asymptotic series for the osculatory cusp changes depending on the leading-order term of the boundary functions $f_{1}(s)$ and $f_{2}(s)$, and is strictly greater than 0 . The numerical evidence from Figure 14 indeed indicates that $C_{1}$ is not zero for osculatory cusps and that the asymptotic behavior depends on $f_{1}(s)$ and $f_{2}(s)$, and not just on the difference $f_{1}(s)-f_{2}(s)$. This conjecture on the unknown constant $C_{1}$ in (35), obtained from numerical investigation, can guide future analytical study of this case.

\subsection{Open problem 2: infinite-curvature cusp with supplementary contact angles.} Another open problem on the singular behavior of the Laplace-Young equation in a cusp domain is the infinite-curvature boundary cusp (i.e., $\lim _{x \rightarrow 0} f_{1}^{\prime \prime}(x)=\infty$ or $\lim _{x \rightarrow 0} f_{2}^{\prime \prime}(x)=\infty$ ) with supplementary contact angles (i.e., $\gamma_{1}+\gamma_{2}=\pi$ ). It was 


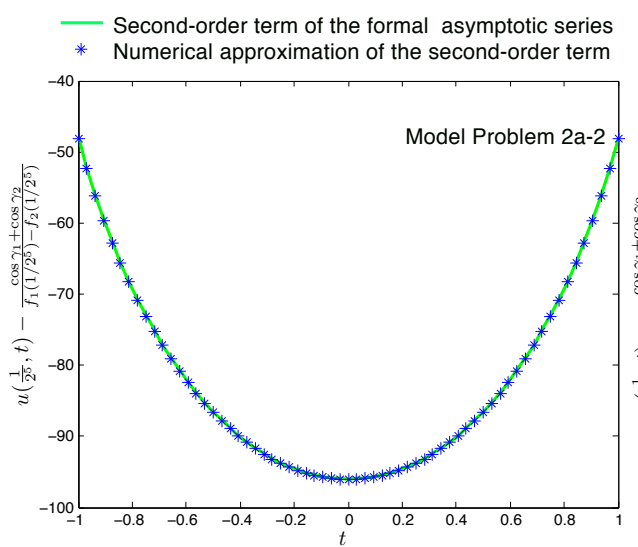

(a) Regular Cusp: MP 2a-2

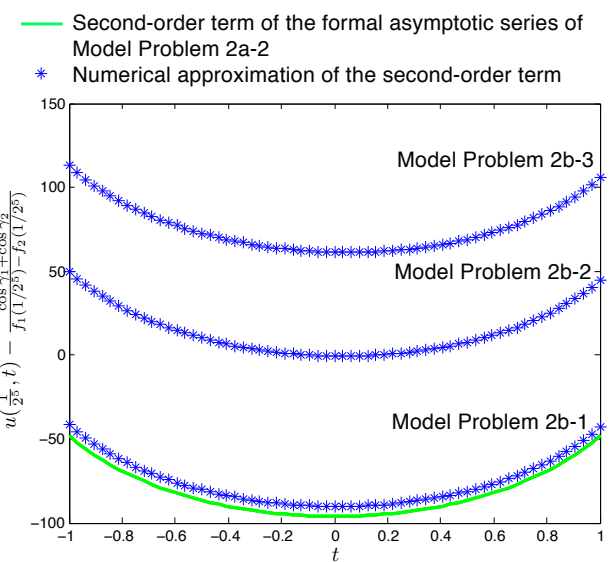

(b) Osculatory Cusp: MP 2b

Figure 14. MPs 2a-2 (unbounded cusp solution) and 2b (unbounded osculatory cusp solution, open problem). FVEM solutions on an $(s, t)$-type grid (Figure $7($ a)) with $33 \times 65$ nodes, with change of variable. The plots show vertical cross sections at $s=1 / 2^{5}$. The left panel shows how the numerical solution tracks the second-order term of the formal asymptotic series. The right panel supports the conjecture that $C_{1}>0$ in (35).

proven in [Aoki and Siegel 2012] that the cusp solution is bounded if the contact angles are supplementary angles and the boundaries forming the cusp have finite curvatures (but it is unbounded if the contact angles are not supplementary).

We conduct numerical experiments for MP 2c (infinite curvature cusp) without change of variable. Lemma 2.1 of [Aoki and Siegel 2012] gives that the solutions of MPs $2 c-1$ and 2c-3 are unbounded, and MP 2c-2 is the supplementary contact angle case with unknown behavior.

As can be seen in Figure 15, the numerical solution surface is bounded if the contact angles are supplementary for this case, where the boundaries forming a cusp have infinite curvature at the cusp. We have conducted various other numerical experiments; however, we were not able to find any evidence of unbounded solutions if the contact angles are supplementary angles. Guided by these numerical results we conjecture that the solution of the Laplace-Young equation in a domain with a cusp is always bounded if the contact angles of the boundaries forming the cusp are supplementary angles. We also note that, as an additional check on the validity of our numerical approach, we have conducted further numerical experiments with cusps with finite curvature boundaries and with the same contact angles as MPs 2c, 


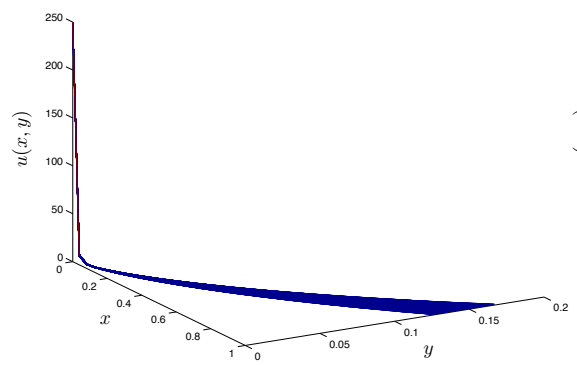

(a) MP 2c-1: $\gamma_{1}+\gamma_{2}=\pi-\frac{1}{180} \pi$

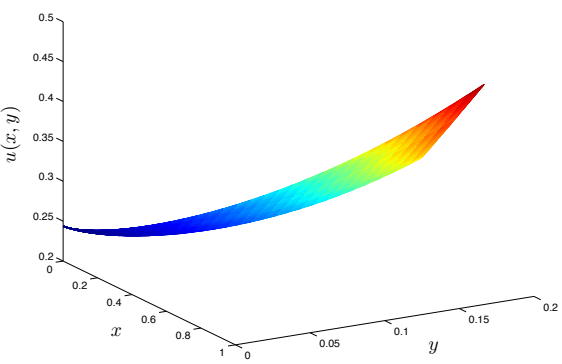

(b) MP 2c-2: $\gamma_{1}+\gamma_{2}=\pi$ (supplementary angles)

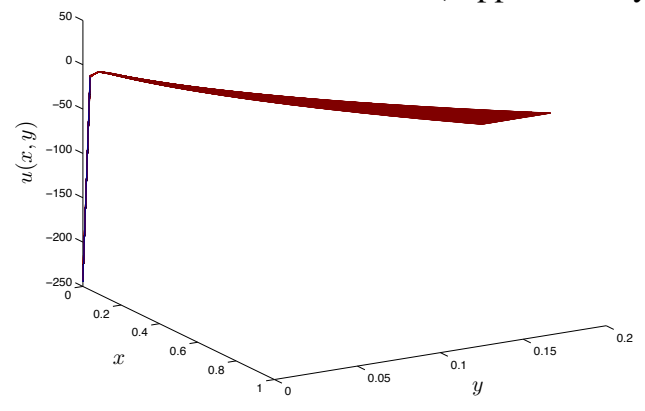

(c) MP 2c-3: $\gamma_{1}+\gamma_{2}=\pi+\frac{1}{180} \pi$

Figure 15. MPs 2c-1, 2c-2, and 2c-3 (infinite curvature cusp). It is known that the solutions for $2 \mathrm{c}-1$ and $2 \mathrm{c}-3$ are unbounded, but the behavior for $2 \mathrm{c}-2$ is an open problem. FVEM solutions on an $(s, t)$-type grid (Figure $7($ a)) with $33 \times 65$ nodes, with change of variable. Surface plots of the capillary surfaces are shown. The numerical result for MP 2c-2 supports the conjecture that the solution is bounded in this case.

and we have confirmed numerically the theoretical prediction that the solution is bounded for supplementary contact angles, and unbounded otherwise.

To conclude, we conjecture that the capillary surface in a cusp domain is bounded if the contact angles of the boundaries forming the cusp are supplementary angles, even if the curvatures of the boundaries are infinite. This conjecture on the open problem of the asymptotic behavior of capillary surfaces in domains with a cusp and supplementary contact angles, obtained from numerical investigation, can guide further analytical study of this case.

\section{Conclusion}

We have proposed a methodology for the numerical study of unbounded capillary surfaces in domains with a sharp corner or a cusp. The methodology was developed 
by incorporating knowledge obtained from asymptotic analysis into a finite element based approximation method. It contains two simple but important ingredients that are combined with the finite volume element method (FVEM) [Bank and Rose 1987; Aoki and De Sterck 2011] or the Galerkin finite element method (FEM) [Strang and Fix 1973; Brenner and Scott 1994]. The first ingredient is to consider a change of variable, with the new solution variable being smoother than the capillary height variable and more amenable to accurate numerical approximation. The second ingredient is to solve the PDE numerically in a new coordinate system that is inspired by asymptotic analysis work, which allows us to accurately represent the discontinuous behavior of the new solution variable at the singular point. We have shown in extensive numerical tests in domains with a sharp corner or a cusp that this approach leads to a global approximation method for singular solutions of the Laplace-Young equation that recovers the proper asymptotic behavior, and is more accurate and has better convergence properties than numerical methods that were considered for singular capillary surfaces before [Scott et al. 2005]. Although we have only considered the Laplace-Young equation and its steep slope approximation, it is likely that the methodology we have proposed can also be useful for other nonlinear elliptic PDEs with singularities. One important limitation of our approach is that in its present form it only works for problems with one singular point. Extension to problems with multiple singular points is a subject for further research.

The main mathematical contribution of this paper is that we were able to formulate conjectures for two open problems on the asymptotic behavior of capillary surfaces in domains with a cusp. These conjectures are derived from numerical investigation of these open problems using the numerical methodology we propose, and they may guide future analytical work on these open problems.

\section{Acknowledgement}

The authors would like to thank Professor Chad Westphal for inspiring discussions, especially for his idea on log-log plots for the horizontal cross-sections of the solution surfaces (Figures 9 and 11).

\section{References}

[Aoki 2007] Y. Aoki, “Analysis of asymptotic solutions for cusp problems in capillarity”, Master's thesis, University of Waterloo, 2007, http://hdl.handle.net/10012/3352.

[Aoki and De Sterck 2011] Y. Aoki and H. De Sterck, "Augmented high order finite volume element method for elliptic PDEs in non-smooth domains: convergence study", J. Comput. Appl. Math. 235:17 (2011), 5177-5187. MR 2012m:65377 Zbl 1221.65299

[Aoki and Siegel 2012] Y. Aoki and D. Siegel, "Bounded and unbounded capillary surfaces in a cusp domain”, Pacific J. Math. 257:1 (2012), 143-165. MR 2948462 Zbl 1248.35043 
[Bank and Rose 1987] R. E. Bank and D. J. Rose, "Some error estimates for the box method", SIAM J. Numer. Anal. 24:4 (1987), 777-787. MR 88j:65235 Zbl 0634.65105

[Brenner and Scott 1994] S. C. Brenner and L. R. Scott, The mathematical theory of finite element methods, Texts in Applied Mathematics 15, Springer, New York, 1994. MR 95f:65001 Zbl 0804.65101

[Concus and Finn 1970] P. Concus and R. Finn, "On a class of capillary surfaces", J. Analyse Math. 23 (1970), 65-70. MR 42 \#8765 Zbl 0257.76007

[Concus and Finn 1974] P. Concus and R. Finn, "On capillary free surfaces in a gravitational field", Acta Math. 132 (1974), 207-223. MR 58 \#32327c Zbl 0382.76005

[Concus and Finn 1989] P. Concus and R. Finn, "Instability of certain capillary surfaces", Manuscripta Math. 63:2 (1989), 209-213. MR 90d:53009 Zbl 0669.49022

[Finn 1986] R. Finn, Equilibrium capillary surfaces, Grundlehren der Mathematischen Wissenschaften 284, Springer, New York, 1986. MR 88f:49001 Zbl 0583.35002

[Grisvard 1985] P. Grisvard, Elliptic problems in nonsmooth domains, Monographs and Studies in Mathematics 24, Pitman, Boston, 1985. MR 86m:35044 Zbl 0695.35060

[Hornung and Mittelmann 1990] U. Hornung and H. D. Mittelmann, "A finite element method for capillary surfaces with volume constraints”, J. Comput. Phys. 87:1 (1990), 126-136. MR 90m:76005 Zbl 0687.76015

[King et al. 1999] J. R. King, J. R. Ockendon, and H. Ockendon, "The Laplace-Young equation near a corner”, Quart. J. Mech. Appl. Math. 52:1 (1999), 73-97. MR 2000f:76017 Zbl 0932.76012

[Lancaster 2010] K. E. Lancaster, "A proof of the Concus-Finn conjecture”, Pacific J. Math. 247:1 (2010), 75-108. MR 2011m:53011 Zbl 05809032

[Miersemann 1993] E. Miersemann, "Asymptotic expansion at a corner for the capillary problem: the singular case”, Pacific J. Math. 157:1 (1993), 95-107. MR 93m:35039 Zbl 0796.76020

[Moon and Spencer 1961] P. Moon and D. E. Spencer, Field theory handbook, Springer, Berlin, 1961. 2nd ed. in 1988. MR 24 \#B2131 Zbl 0097.39403

[Nigro et al. 2000] N. J. Nigro, B. P. Zellmer, D. Shangguan, and P. S. Lee, "A modified finite element method for determining equilibrium capillary surfaces of liquids with specified volumes", International Journal for Numerical Methods in Fluids 33:6 (2000), 833-846. Zbl 0986.76044

[Norbury et al. 2005] J. Norbury, G. C. Sander, and C. F. Scott, "Corner solutions of the LaplaceYoung equation”, Quart. J. Mech. Appl. Math. 58:1 (2005), 55-71. MR 2006b:76017 Zbl 1064.76020

[Polevikov 1999] V. K. Polevikov, "Methods for the numerical modeling of equilibrium capillary surfaces”, Differ. Uravn. 35:7 (1999), 975-981. MR 2000k:76099 Zbl 1064.76579

[Polevikov 2004] V. K. Polevikov, "Methods for numerical modeling of two-dimensional capillary surfaces”, Comput. Methods Appl. Math. 4:1 (2004), 66-93. MR 2005i:76045 Zbl 1221.76128

[Scholz 2001] M. Scholz, Über das Verhalten von Kapillarflächen in Spitzen, Ph.D. thesis, Universität Leipzig, 2001, http://www.people.imise.uni-leipzig.de/markus.scholz/pdf/p1.pdf.

[Scholz 2004] M. Scholz, "On the asymptotic behaviour of capillary surfaces in cusps", Z. Angew. Math. Phys. 55:2 (2004), 216-234. MR 2005e:76023 Zbl 1058.35059

[Scott et al. 2005] C. F. Scott, G. C. Sander, and J. Norbury, "Computation of capillary surfaces for the Laplace-Young equation”, Quart. J. Mech. Appl. Math. 58:2 (2005), 201-212. MR 2006a:76019 Zbl 1072.76014

[Simon 1980] L. Simon, "Regularity of capillary surfaces over domains with corners", Pacific J. Math. 88:2 (1980), 363-377. MR 82d:49043 Zbl 0467.35022 
[Strang and Fix 1973] G. Strang and G. J. Fix, An analysis of the finite element method, Prentice-Hall, Englewood Cliffs, NJ, 1973. MR 56 \#1747 Zbl 0356.65096

Received June 13, 2012. Revised September 18, 2012.

\section{YASUNORI AOKI}

DEPARTMENT OF APPLIED MATHEMATICS

UNIVERSITY OF WATERLOO

200 UnIVERSITY AVE. WeSt

WATERLOO N2L 3G1

CANADA

yaoki@uwaterloo.ca

HANS De STERCK

Department of ApPlied Mathematics

UNIVERSITY OF WATERLOO

200 UniVERsity AVE. West

WATERLOO N2L 3G1

CANADA

hdesterck@uwaterloo.ca 


\title{
PACIFIC JOURNAL OF MATHEMATICS
}

\author{
msp.org/pjm
}

Founded in 1951 by E. F. Beckenbach (1906-1982) and F. Wolf (1904-1989)

\section{EDITORS}

V. S. Varadarajan (Managing Editor)

Department of Mathematics

University of California

Los Angeles, CA 90095-1555

pacific@math.ucla.edu

Paul Balmer

Department of Mathematics

University of California

Los Angeles, CA 90095-1555

balmer@math.ucla.edu

Daryl Cooper

Department of Mathematics

University of California

Santa Barbara, CA 93106-3080 cooper@math.ucsb.edu

Jiang-Hua $\mathrm{Lu}$

Department of Mathematics

Pokfulam Rd., Hong Kong jhlu@maths.hku.hk
The University of Hong Kong

Don Blasius

Department of Mathematics University of California

Los Angeles, CA 90095-1555

blasius@math.ucla.edu

Robert Finn

Department of Mathematics Stanford University

Stanford, CA 94305-2125

finn@math.stanford.edu

Sorin Popa

Department of Mathematics

University of California

Los Angeles, CA 90095-1555 popa@math.ucla.edu

Paul Yang

Department of Mathematics Princeton University

Princeton NJ 08544-1000

yang@math.princeton.edu

\section{PRODUCTION}

Silvio Levy, Scientific Editor, production@msp.org

\section{SUPPORTING INSTITUTIONS}

ACADEMIA SINICA, TAIPEI

CALIFORNIA INST. OF TECHNOLOGY

INST. DE MATEMÁTICA PURA E APLICADA

KEIO UNIVERSITY

MATH. SCIENCES RESEARCH INSTITUTE

NEW MEXICO STATE UNIV.

OREGON STATE UNIV.

\author{
STANFORD UNIVERSITY \\ UNIV. OF BRITISH COLUMBIA \\ UNIV. OF CALIFORNIA, BERKELEY \\ UNIV. OF CALIFORNIA, DAVIS \\ UNIV. OF CALIFORNIA, LOS ANGELES \\ UNIV. OF CALIFORNIA, RIVERSIDE \\ UNIV. OF CALIFORNIA, SAN DIEGO \\ UNIV. OF CALIF., SANTA BARBARA
}

\author{
Vyjayanthi Chari \\ Department of Mathematics \\ University of California \\ Riverside, CA 92521-0135 \\ chari@math.ucr.edu \\ Kefeng Liu \\ Department of Mathematics \\ University of California \\ Los Angeles, CA 90095-1555 \\ liu@math.ucla.edu \\ Jie Qing \\ Department of Mathematics \\ University of California \\ Santa Cruz, CA 95064 \\ qing@cats.ucsc.edu
}

These supporting institutions contribute to the cost of publication of this Journal, but they are not owners or publishers and have no responsibility for its contents or policies.

See inside back cover or msp.org/pjm for submission instructions.

The subscription price for 2014 is US \$410/year for the electronic version, and \$535/year for print and electronic.

Subscriptions, requests for back issues and changes of subscribers address should be sent to Pacific Journal of Mathematics, P.O. Box 4163, Berkeley, CA 94704-0163, U.S.A. The Pacific Journal of Mathematics is indexed by Mathematical Reviews, Zentralblatt MATH, PASCAL CNRS Index, Referativnyi Zhurnal, Current Mathematical Publications and Web of Knowledge (Science Citation Index).

The Pacific Journal of Mathematics (ISSN 0030-8730) at the University of California, c/o Department of Mathematics, 798 Evans Hall \#3840, Berkeley, CA 94720-3840, is published twelve times a year. Periodical rate postage paid at Berkeley, CA 94704, and additional mailing offices. POSTMASTER: send address changes to Pacific Journal of Mathematics, P.O. Box 4163, Berkeley, CA 94704-0163.

PJM peer review and production are managed by EditFLOW ${ }^{\circledR}$ from Mathematical Sciences Publishers.

\section{PUBLISHED BY}

mathematical sciences publishers

nonprofit scientific publishing

http://msp.org/

(C) 2014 Mathematical Sciences Publishers 


\section{PACIFIC JOURNAL OF MATHEMATICS}

Volume $267 \quad$ No. $1 \quad$ January 2014

Numerical study of unbounded capillary surfaces

YASUNORI AOKI and HANS DE STERCK

Dual $R$-groups of the inner forms of $\operatorname{SL}(N)$

KUOK FAI CHAO and WEN-WEI LI

Automorphisms and quotients of quaternionic fake quadrics

AMIR DŽAmBIĆ and XAVIER ROULLEAU

Distance of bridge surfaces for links with essential meridional spheres 121

YEONHEE JANG

Normal states of type III factors

YASUYUKI KAWAHIGASHI, YOSHIKO OGATA and ERLING

STØRMER

Eigenvalues and entropies under the harmonic-Ricci flow

YI LI

Quantum extremal loop weight modules and monomial crystals

MATHIEU MANSUY

Lefschetz fibrations with small slope

NAOYUKI MONDEN 